\title{
Families of hyperfinite subfactors with the same standard invariant and prescribed fundamental group
}

\author{
Arnaud Brothier and Stefaan Vaes*
}

\begin{abstract}
We construct irreducible hyperfinite subfactors of index 6 with a prescribed fundamental group from a large family containing all countable and many uncountable subgroups of $\mathbb{R}_{+}$. We also prove that there are unclassifiably many irreducible hyperfinite group-type subfactors of index 6 that all have the same standard invariant. More precisely, we associate such a subfactor to every ergodic measure preserving automorphism of the interval $[0,1]$ and prove that the resulting subfactors are isomorphic if and only if the automorphisms are conjugate.
\end{abstract}

Mathematics Subject Classification (2010). 46L37; 46L36, 46L55.

Keywords. Subfactor, standard invariant, von Neumann algebra, deformation/rigidity theory.

\section{Introduction and statement of the main results}

To every inclusion of $\mathrm{II}_{1}$ factors $N \subset M$ with finite Jones index [18] is associated a group-like object $\mathcal{G}_{N \subset M}$ called the standard invariant. In [25], Popa proved the fundamental result that every strongly amenable standard invariant arises from precisely one hyperfinite subfactor. When the standard invariant is nonamenable, much less is known. It is for instance wide open to decide at which index values larger than 4 , the $A_{\infty}$ Temperley-Lieb-Jones standard invariant arises from a hyperfinite subfactor, and if it does, whether this subfactor is unique or not.

In [3], Bisch and Haagerup associated to every countable group $\Gamma$ generated by finite subgroups $H, K \subset \Gamma$ and to every outer action $\left(\alpha_{g}\right)_{g \in \Gamma}$ of $\Gamma$ on the hyperfinite $\mathrm{II}_{1}$ factor $R$, the group-type subfactor $S(\alpha): R^{H} \subset R \rtimes K$. This construction gives rise to a wealth of infinite depth subfactors with different types of properties (amenable vs. strongly amenable, property (T), etc). Popa proved in [26] a deep cocycle superrigidity theorem for Connes-Størmer Bernoulli actions of infinite property (T) groups $\Gamma$ and used this to show that all these groups $\Gamma$ admit

\footnotetext{
*Funded by ERC Consolidator Grant 614195 from the European Research Council under the European Union's Seventh Framework Programme, and by Research Programme G.0639.11 of the Research Foundation - Flanders (FWO).
} 


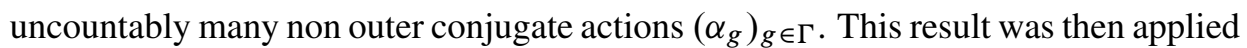
in [4] to property ( $\mathrm{T}$ ) groups $\Gamma$ generated by subgroups $H \cong \mathbb{Z} / 2 \mathbb{Z}$ and $K \cong \mathbb{Z} / 3 \mathbb{Z}$ and implied that the resulting subfactors $S(\alpha)$ are nonisomorphic, but nevertheless all have the same standard invariant.

Also amplifications can give rise to nonisomorphic subfactors with the same standard invariant. If $N \subset M$ is a subfactor and $t>0$, the amplification $(N \subset M)^{t}$ is defined as follows : choose a projection $p \in M_{n}(\mathbb{C}) \otimes N$ with $(\operatorname{Tr} \otimes \tau)(p)=t$ and define $(N \subset M)^{t}$ as the inclusion $p\left(M_{n}(\mathbb{C}) \otimes N\right) p \subset p\left(M_{n}(\mathbb{C}) \otimes M\right) p$. Following [24, Definition 5.4.7], the relative fundamental group of the subfactor $N \subset M$ is then defined as

$$
\mathcal{F}(N \subset M)=\left\{t>0 \mid(N \subset M)^{t} \cong(N \subset M)\right\}
$$

and is a subgroup of $\mathbb{R}_{+}$. For the group-type subfactors $S(\alpha): R^{H} \subset R \rtimes K$, it is shown in [4] that $\mathcal{F}(N \subset M)$ is a subgroup of the fundamental group $\mathcal{F}(\alpha)$ introduced in [26]. Since it is proven in [26] that the noncommutative Bernoulli actions of an infinite property $(\mathrm{T})$ group have trivial fundamental group, the resulting subfactors $S(\alpha)$ also have trivial relative fundamental group and the amplifications $S(\alpha)^{t}, t>0$, form an uncountable family of nonisomorphic subfactors with the same standard invariant.

In Theorem A below, we refine the above results and show that there are "unclassifiably" many nonisomorphic subfactors of index 6 with the same standard invariant. More precisely, to every ergodic measure preserving automorphism $\Delta$ of the interval $[0,1]$, we associate an outer action $\alpha^{\Delta}$ of the modular group $\operatorname{PSL}(2, \mathbb{Z})=\mathbb{Z} / 2 \mathbb{Z} * \mathbb{Z} / 3 \mathbb{Z}$ on the hyperfinite $\mathrm{II}_{1}$ factor $R$ and consider the corresponding subfactor $S\left(\alpha^{\Delta}\right): R^{\mathbb{Z} / 2 \mathbb{Z}} \subset R \rtimes \mathbb{Z} / 3 \mathbb{Z}$. All these subfactors $S\left(\alpha^{\Delta}\right)$ have index 6 and the same standard invariant $\mathcal{G}$. We prove that the subfactor $S\left(\alpha^{\Delta}\right)$ is isomorphic with $S\left(\alpha^{\Delta^{\prime}}\right)$ if and only if $\Delta$ is conjugate to $\Delta^{\prime}$, meaning that $\Delta^{\prime}=\theta \circ \Delta \circ \theta^{-1}$ for some measure preserving transformation $\theta$. Since the classification of ergodic transformations up to conjugacy is wild in any possible sense (see e.g. $[14,11]$ ), the classification of hyperfinite index 6 subfactors with standard invariant $\mathcal{G}$ is at least as wild.

In Theorem A, we also construct outer actions $\alpha$ of the modular group PSL $(2, \mathbb{Z})$ such that the resulting subfactor $S(\alpha)$ has any prescribed relative fundamental group from the large family $\mathcal{S}$ of subgroups of $\mathbb{R}_{+}$studied in [31, Section 2]. This family $\mathcal{S}$ contains all countable subgroups of $\mathbb{R}_{+}$, as well as many uncountable subgroups that can have any Hausdorff dimension between 0 and 1 .

Note that the main result of [31] showed that all groups in the family $\mathcal{S}$ arise as the fundamental group $\mathcal{F}(M)$ of a $\mathrm{II}_{1}$ factor $M$ with separable predual. The result in [31] is an existence theorem that ultimately relies on a Baire category argument. Explicit examples of $\mathrm{II}_{1}$ factors with prescribed fundamental group in $\mathcal{S}$ were constructed in [9]. In Corollary 4.4 below, we also give a new and explicit proof of that result, using Theorem $A$ and the main results of $[32,33]$. 
Theorem A. Let $n \geq 2$ be an integer and $m \geq 3$ a prime number. Put $\Gamma=(\mathbb{Z} / n \mathbb{Z}) *(\mathbb{Z} / m \mathbb{Z})$. For every outer action $\left(\alpha_{g}\right)_{g \in \Gamma}$ of $\Gamma$ on the hyperfinite $\mathrm{II}_{1}$ factor, consider the associated group-type subfactor $S(\alpha): R^{\mathbb{Z} / n \mathbb{Z}} \subset R \rtimes \mathbb{Z} / m \mathbb{Z}$. Note that these subfactors are irreducible, have index $\mathrm{nm}$ and have a standard invariant that only depends on the integers $n$ and $m$.

(1) For every group $H \in \mathcal{S}$, there exists an outer action $\left(\alpha_{g}\right)_{g \in \Gamma}$ on the hyperfinite $\mathrm{II}_{1}$ factor $R$ such that the subfactor $S(\alpha)$ has relative fundamental group $H$.

(2) To every ergodic probability measure preserving automorphism $\Delta$ of a standard nonatomic probability space, we can associate an outer action $\left(\alpha_{g}^{\Delta}\right)_{g \in \Gamma}$ on $R$ such that the corresponding subfactors $S\left(\alpha^{\Delta}\right)$ are isomorphic if and only if the automorphisms are conjugate.

As mentioned above, classifying group-type subfactors $R^{H} \subset R \rtimes K$ is closely related to classifying actions up to outer/cocycle conjugacy. Two outer actions $\alpha$ and $\beta$ of a countable group $\Gamma$ on a $\mathrm{II}_{1}$ factor $M$ are called outer conjugate if there exist automorphisms $\psi \in \operatorname{Aut}(M), \delta \in \operatorname{Aut}(\Gamma)$ and a family of unitaries $\left(w_{g}\right)_{g \in \Gamma}$ in $M$ such that $\beta_{\delta(g)} \circ \psi=\psi \circ\left(\operatorname{Ad} w_{g}\right) \circ \alpha_{g}$ for all $g \in \Gamma$. If the unitaries $w_{g}$ can be chosen in such a way that $w_{g h}=w_{g} \alpha_{g}\left(w_{h}\right)$ for all $g, h \in \Gamma$, then $\alpha$ and $\beta$ are called cocycle conjugate.

There are several parallels between the study of outer actions of $\Gamma$ on the hyperfinite $\mathrm{II}_{1}$ factor $R$ up to cocycle conjugacy and the study of free ergodic probability measure preserving (pmp) actions $\Gamma \curvearrowright(X, \mu)$ up to orbit equivalence. Recall for instance that by [22] all free ergodic pmp actions of an infinite amenable group $\Gamma$ are orbit equivalent, while it was shown in [20] that all outer actions of an amenable group $\Gamma$ on $R$ are cocycle conjugate.

Nonamenable groups $\Gamma$ admit uncountably many non orbit equivalent actions: this was first proven for the free groups $\mathbb{F}_{n}$ in [13] and then for groups containing a copy of $\mathbb{F}_{2}$ in [16], and finally in the general case in [10]. This last result is based on [12], where it is shown that every nonamenable group $\Gamma$ contains $\mathbb{F}_{2}$ "measurably". In particular, there is no explicit construction of an uncountable family of non orbit equivalent actions of an arbitrary nonamenable group $\Gamma$, but rather a proof of their existence. Quite surprisingly, our Theorem B below provides an explicit and rather easy uncountable family of non outer conjugate actions of an arbitrary nonamenable group $\Gamma$ on the hyperfinite $\mathrm{II}_{1}$ factor. Note here that it was already proven in [19] that every nonamenable group $\Gamma$ admits at least two non outer conjugate actions on the hyperfinite $\mathrm{II}_{1}$ factor, while it was shown in [26] that every

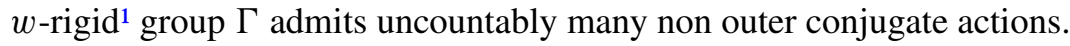

\footnotetext{
${ }^{1} \mathrm{~A}$ countable group $\Gamma$ is called $w$-rigid if $\Gamma$ admits an infinite normal subgroup with the relative property $(\mathrm{T})$ of Kazhdan-Margulis.
} 
Theorem B. Let $\Gamma$ be any nonamenable group and let $\Lambda$ be any amenable group that has a torsion free FC-radical ${ }^{2}$, e.g. take $\Lambda$ to be an amenable icc group, or an amenable torsion free group. Realize the hyperfinite $\mathrm{II}_{1}$ factor $R$ as

$$
R=\left(M_{2}(\mathbb{C})^{\Gamma \times \Lambda} \bar{\otimes} M_{2}(\mathbb{C})^{\Lambda}\right) \rtimes \Lambda
$$

where $\Lambda$ acts diagonally by Bernoulli shifts and where we take infinite tensor products with respect to the trace on $M_{2}(\mathbb{C})$. The Bernoulli shift of $\Gamma$ yields an outer action $\left(\alpha_{g}^{\Lambda}\right)_{g \in \Gamma}$ of $\Gamma$ on $R$.

The actions $\left(\alpha_{g}^{\Lambda_{1}}\right)_{g \in \Gamma}$ and $\left(\alpha_{g}^{\Lambda_{2}}\right)_{g \in \Gamma}$ are outer conjugate if and only if the groups $\Lambda_{1}, \Lambda_{2}$ are isomorphic.

We prove Theorems A and B by using Popa's deformation/rigidity methods, in particular the spectral gap rigidity of [29] and the malleable deformation for Bernoulli actions of $[26,28]$.

Our methods can best be explained for the very explicit actions $\left(\alpha_{g}^{\Lambda}\right)_{g \in \Gamma}$ of the arbitrary nonamenable group $\Gamma$ on the hyperfinite $\mathrm{II}_{1}$ factor

$$
M(\Lambda)=\left(M_{2}(\mathbb{C})^{\Gamma \times \Lambda} \bar{\otimes} M_{2}(\mathbb{C})^{\Lambda}\right) \rtimes \Lambda
$$

as defined in Theorem B. Contrary to the approach in [26], we cannot expect to prove a general cocycle superrigidity theorem for $\left(\alpha_{g}^{\Lambda}\right)_{g \in \Gamma}$, because $\Gamma$ might be the free group, or a free product group, and such groups do not have cocycle superrigid actions.

So we need to use another method to prove that every outer conjugacy $\psi$ : $M\left(\Lambda_{1}\right) \rightarrow M\left(\Lambda_{2}\right)$ between $\left(\alpha_{g}^{\Lambda_{1}}\right)_{g \in \Gamma}$ and $\left(\alpha_{g}^{\Lambda_{2}}\right)_{g \in \Gamma}$ is actually a conjugacy up to an inner automorphism. For this, note that by construction, the subalgebra $P_{i}=M_{2}(\mathbb{C})^{\Lambda_{i}} \rtimes \Lambda_{i}$ of $M\left(\Lambda_{i}\right)$ is pointwise fixed under the action $\left(\alpha_{g}^{\Lambda_{i}}\right)_{g \in \Gamma}$. Using the methods of $[26,28,29]$, including a malleable deformation of $M\left(\Lambda_{2}\right)$ and spectral gap rigidity coming from the nonamenability of $\Gamma$, we deduce that the deformation converges uniformly to the identity on the unit ball of $\psi\left(P_{1}\right)$ and find a unitary $w \in M\left(\Lambda_{2}\right)$ such that $w \psi\left(P_{1}\right) w^{*}=P_{2}$. Replacing $\psi$ by $(\operatorname{Ad} w) \circ \psi$, we may assume that $\psi\left(P_{1}\right)=P_{2}$. Still, $\psi$ is an outer conjugacy between $\left(\alpha_{g}^{\Lambda_{1}}\right)_{g \in \Gamma}$ and

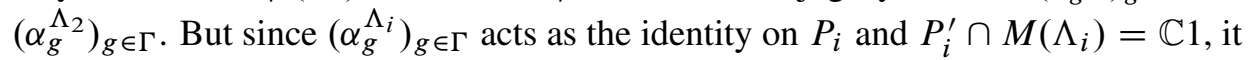

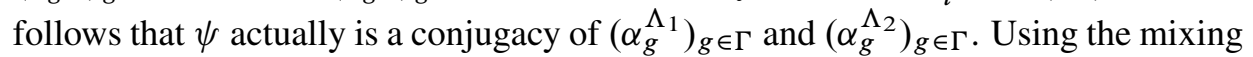
techniques of [28, Section 3], we then finally deduce that $\psi$ must send $M_{2}(\mathbb{C})^{\Lambda_{1}}$ onto $M_{2}(\mathbb{C})^{\Lambda_{2}}$. Since also $\psi\left(P_{1}\right)=P_{2}$, the actions $\Lambda_{i} \curvearrowright M_{2}(\mathbb{C})^{\Lambda_{i}}$ follow cocycle conjugate and, in particular, $\Lambda_{1} \cong \Lambda_{2}$.

Acknowledgements. We are very grateful to Darren Creutz and Cesar E. Silva for their advice on rank one ergodic transformations (see 4.1 below).

\footnotetext{
${ }^{2}$ The FC-radical of a countable group $\Gamma$ is the normal subgroup that consists of all elements of $\Gamma$ that have a finite conjugacy class.
} 


\section{Preliminaries}

We start by recalling Popa's theory of intertwining-by-bimodules developed in [28, Section 2]. Let $(M, \tau)$ be a von Neumann algebra with separable predual equipped with a normal faithful tracial state. Let $P, Q \subset M$ be von Neumann subalgebras. Following [28], write $P \prec_{M} Q$ if there exist projections $p \in P, q \in Q$, a normal unital $*$-homomorphism $\theta: p P p \rightarrow q Q q$ and a nonzero partial isometry $v \in p M q$ satisfying $a v=v \theta(a)$ for all $a \in P$. By [28, Corollary 2.3], we have $P \nprec_{M} Q$ if and only if there exists a sequence of unitaries $v_{n} \in \mathcal{U}(P)$ satisfying $\lim _{n}\left\|E_{Q}\left(a v_{n} b\right)\right\|_{2}=0$ for all $a, b \in M$.

Also recall that a trace preserving action $\left(\gamma_{s}\right)_{s \in \Lambda}$ of a countable group $\Lambda$ on a von Neumann algebra $(B, \tau)$ with normal faithful tracial state $\tau$ is called mixing if for all $a, b \in B$ with $\tau(a)=0=\tau(b)$, we have $\lim _{s \rightarrow \infty} \tau\left(\gamma_{s}(a) b\right)=0$.

Our first lemma provides a variant of the results in [28, Section 3]. For completeness, we provide a complete proof.

Lemma 2.1. Let $(B, \tau)$ and $(D, \operatorname{Tr})$ be von Neumann algebras equipped with normal faithful traces with $\tau(1)=1$ and with $\mathrm{Tr}$ being finite or semifinite. Assume that a countable group $\Lambda$ acts in a trace preserving way on $(B, \tau)$ and $(D, \operatorname{Tr})$. Denote these actions, as well as their diagonal product on $B \bar{\otimes} D$, by $\left(\gamma_{s}\right)_{s \in \Lambda}$. Assume that the action $\Lambda \curvearrowright(B, \tau)$ is mixing.

Let $p \in D$ be a projection with $\operatorname{Tr}(p)<\infty$. Denote $M=p((B \bar{\otimes} D) \rtimes \Lambda) p$ and $P=p(D \rtimes \Lambda) p$. If $Q \subset P$ is a von Neumann subalgebra with $Q \nprec_{P} p D p$ and if $v \in M$ satisfies $v Q \subset P v$, then $v \in P$.

Proof. By assumption, we get a sequence $w_{n} \in \mathcal{U}(Q)$ satisfying $\lim _{n}\left\|E_{p D p}\left(x w_{n} y\right)\right\|_{2}$ $=0$ for all $x, y \in P$. Denote by $\left(u_{s}\right)_{s \in \Lambda}$ the canonical unitaries in the crossed product $D \rtimes \Lambda$. Every $w_{n}$ has a Fourier decomposition

$$
w_{n}=\sum_{s \in \Lambda}\left(w_{n}\right)_{s} u_{s} \quad \text { with }\left(w_{n}\right)_{s} \in p D \gamma_{s}(p)
$$

We claim that for every fixed $s \in \Lambda$, we have $\lim _{n}\left\|\left(w_{n}\right)_{s}\right\|_{2}=0$. To prove this claim, fix $s \in \Lambda$. For every unitary $v \in \mathcal{U}(D)$, we have the element $p u_{s}^{*} v p \in P$ and therefore

$$
\lim _{n}\left\|E_{p D p}\left(w_{n} p u_{s}^{*} v p\right)\right\|_{2}=0 .
$$

Since $E_{p D p}\left(w_{n} p u_{s}^{*} v p\right)=\left(w_{n}\right)_{s} \gamma_{s}(p) v p=\left(w_{n}\right)_{s} v p$, we get that $\lim _{n}\left\|\left(w_{n}\right)_{s} v p\right\|_{2}$ $=0$. Then also $\lim _{n}\left\|\left(w_{n}\right)_{s} v p v^{*}\right\|_{2}=0$. Since the join of all the projections $v p v^{*}$, $v \in \mathcal{U}(D)$, equals the central support $z \in \mathcal{Z}(D)$ of $p \in D$, we get that $\lim _{n}\left\|\left(w_{n}\right)_{s} z\right\|_{2}=0$. But $\left(w_{n}\right)_{s} z=z\left(w_{n}\right)_{s}=\left(w_{n}\right)_{s}$ and the claim is proven.

We next prove that

$$
\lim _{n}\left\|E_{P}\left(x w_{n} y\right)\right\|_{2}=0 \text { for all } x, y \in M \ominus P .
$$


Since the linear span of $P(B \ominus \mathbb{C} 1)$ is $\|\cdot\|_{2}$-dense in $M \ominus P$, it suffices to prove (2.1) for $x, y \in B \ominus \mathbb{C} 1$. But then

$$
E_{P}\left(x w_{n} y\right)=\sum_{s \in \Lambda} \tau\left(x \gamma_{s}(y)\right)\left(w_{n}\right)_{s} u_{s}
$$

It follows that

$$
\left\|E_{P}\left(x w_{n} y\right)\right\|_{2}^{2}=\sum_{s \in \Lambda}\left|\tau\left(x \gamma_{s}(y)\right)\right|^{2}\left\|\left(w_{n}\right)_{s}\right\|_{2}^{2} .
$$

Fix $\varepsilon>0$. Since the action $\Lambda \curvearrowright(B, \tau)$ is mixing, take a finite subset $\mathcal{F} \subset \Lambda$ such that $\left|\tau\left(x \gamma_{s}(y)\right)\right|^{2}<\varepsilon / \operatorname{Tr}(p)$ for every $s \in \Lambda-\mathcal{F}$. Since $\lim _{n}\left\|\left(w_{n}\right)_{s}\right\|_{2}=0$ for every fixed $s \in \Lambda$, we next take $n_{0}$ such that

$$
\sum_{s \in \mathcal{F}}\left|\tau\left(x \gamma_{s}(y)\right)\right|^{2}\left\|\left(w_{n}\right)_{s}\right\|_{2}^{2}<\varepsilon \quad \text { for all } n \geq n_{0} .
$$

We conclude that for all $n \geq n_{0}$,

$$
\begin{aligned}
\left\|E_{P}\left(x w_{n} y\right)\right\|_{2}^{2} & \leq \varepsilon+\sum_{s \in \Lambda-\mathcal{F}}\left|\tau\left(x \gamma_{s}(y)\right)\right|^{2}\left\|\left(w_{n}\right)_{s}\right\|_{2}^{2} \\
& \leq \varepsilon+\frac{\varepsilon}{\operatorname{Tr}(p)} \sum_{s \in \Lambda-\mathcal{F}}\left\|\left(w_{n}\right)\right\|_{2}^{2} \leq \varepsilon+\frac{\varepsilon}{\operatorname{Tr}(p)}\left\|w_{n}\right\|_{2}^{2}=2 \varepsilon .
\end{aligned}
$$

So (2.1) is proven. The conclusion of the lemma now follows from [35, Lemma D.3].

Let $(M, \tau)$ be a tracial von Neumann algebra. Recall from [23, Section 1.2] that von Neumann subalgebras $M_{1}, M_{2} \subset M$ are said to form a commuting square when $E_{M_{1}} \circ E_{M_{2}}=E_{M_{1} \cap M_{2}}=E_{M_{2}} \circ E_{M_{1}}$. We need the following easy lemma and include a complete proof for the convenience of the reader.

Lemma 2.2. Let $(M, \tau)$ be a tracial von Neumann algebra with von Neumann subalgebras $M_{1}, M_{2} \subset M$ that form a commuting square. Assume that the linear span of $M_{1} M_{2}$ is $\|\cdot\|_{2}$-dense in $M$. If $Q \subset M_{1}$ is a von Neumann subalgebra and $Q \nprec_{M_{1}} M_{1} \cap M_{2}$, then $Q \nprec_{M} M_{2}$.

Proof. Put $P=M_{1} \cap M_{2}$. Assume that $Q \subset M_{1}$ and $Q \nprec_{M_{1}} P$. We then find a sequence of unitaries $w_{n} \in \mathcal{U}(Q)$ satisfying $\lim _{n}\left\|E_{P}\left(x w_{n} y\right)\right\|_{2}=0$ for all $x, y \in M_{1}$. For all $x, y \in M_{1}$ and for all $a, b \in M_{2}$, we have

$$
E_{M_{2}}\left(a x w_{n} y b\right)=a E_{M_{2}}\left(x w_{n} y\right) b=a E_{P}\left(x w_{n} y\right) b .
$$

Therefore, $\lim _{n}\left\|E_{M_{2}}\left(a x w_{n} y b\right)\right\|_{2}=0$. Since the linear span of $M_{1} M_{2}$ is $\|\cdot\|_{2^{-}}$ dense in $M$, we conclude that $\lim _{n}\left\|E_{M_{2}}\left(c w_{n} d\right)\right\|_{2}=0$ for all $c, d \in M$. This implies that $Q \nprec_{M} M_{2}$. 
We finally recall the concept of a co-induced action. Assume that $(B, \tau)$ is a tracial von Neumann algebra with a trace preserving action $\left(\beta_{g}\right)_{g \in \Gamma_{0}}$. Assume that $\Gamma_{0}<\Gamma$. The co-induced action of $\left(\beta_{g}\right)_{g \in \Gamma_{0}}$ to $\Gamma$ is the following action $\left(\alpha_{g}\right)_{g \in \Gamma}$ on the infinite tensor product $(A, \tau)=(B, \tau)^{\Gamma / \Gamma_{0}}$. First choose a section $\theta: \Gamma / \Gamma_{0} \rightarrow \Gamma$ with $\theta\left(e \Gamma_{0}\right)=e$. We then get the 1-cocycle $\omega: \Gamma \times \Gamma / \Gamma_{0} \rightarrow \Gamma_{0}$ determined by

$$
g \theta\left(h \Gamma_{0}\right)=\theta\left(g h \Gamma_{0}\right) \omega\left(g, h \Gamma_{0}\right) \quad \text { for all } g \in \Gamma, h \Gamma_{0} \in \Gamma / \Gamma_{0} .
$$

We denote by $\pi_{h \Gamma_{0}}: B \rightarrow A$ the embedding of $B$ as the $h \Gamma_{0}$-th tensor factor. There is a unique trace preserving action $\left(\alpha_{g}\right)_{g \in \Gamma}$ of $\Gamma$ on $A$ satisfying

$$
\alpha_{g}\left(\pi_{h \Gamma_{0}}(b)\right)=\pi_{g h \Gamma_{0}}\left(\beta_{\omega\left(g, h \Gamma_{0}\right)}(b)\right) \quad \text { for all } g \in \Gamma, h \Gamma_{0} \in \Gamma / \Gamma_{0}, b \in B .
$$

Note that by construction $\alpha_{g}\left(\pi_{e \Gamma_{0}}(b)\right)=\pi_{e \Gamma_{0}}\left(\beta_{g}(b)\right)$ for all $g \in \Gamma_{0}, b \in B$.

\section{A first outer conjugacy lemma and the proof of Theorem B}

Throughout this section, we fix a countable group $\Gamma$ with a subgroup $\Gamma_{0}<\Gamma$ that is not co-amenable, i.e. such that the set $\Gamma / \Gamma_{0}$ does not admit a $\Gamma$-invariant mean. In particular, one can take $\Gamma_{0}=\{e\}$, or $\Gamma_{0}$ amenable, and $\Gamma$ any nonamenable group. We also fix an infinite group $\Lambda$. We let these groups $\Gamma$ and $\Lambda$ act in the following way on von Neumann algebras.

- Let $(B, \tau)$ be a von Neumann algebra equipped with a normal faithful tracial state $\tau$. We assume that $(B, \tau)$ comes with commuting trace preserving faithful ${ }^{3}$ actions $\left(\beta_{g}\right)_{g \in \Gamma_{0}}$ and $\left(\gamma_{s}\right)_{s \in \Lambda}$. We assume that the action $\left(\gamma_{s}\right)_{s \in \Lambda}$ on $(B, \tau)$ is mixing.

- We put $(A, \tau)=(B, \tau)^{\Gamma / \Gamma_{0}}$. As recalled at the end of Section 2, we can define the co-induced action of $\left(\beta_{g}\right)_{g \in \Gamma_{0}}$ and this is an action $\left(\alpha_{g}\right)_{g \in \Gamma}$ of $\Gamma$ on $(A, \tau)$. We also consider the diagonal action of $\Lambda$ on $(A, \tau)$ that we still denote as $\left(\gamma_{s}\right)_{s \in \Lambda}$. Note that $\left(\alpha_{g}\right)_{g \in \Gamma}$ commutes with $\left(\gamma_{s}\right)_{s \in \Lambda}$.

- Let $(D, \operatorname{Tr})$ be a von Neumann algebra equipped with a normal, finite or semifinite, faithful trace Tr. Assume that $\left(\gamma_{s}\right)_{s \in \Lambda}$ is a trace preserving action on $(D, \operatorname{Tr})$ and that one of the following assumptions hold.

(1) $D$ is a factor, the action $\left(\gamma_{s}\right)_{s \in \Lambda}$ is outer and the group $\Lambda$ has a torsion free FC-radical. For instance, we could take $\Lambda$ to be an icc group, or a torsion free group.

(2) $D$ is diffuse abelian and the action $\left(\gamma_{s}\right)_{s \in \Lambda}$ is essentially free and ergodic.

\footnotetext{
${ }^{3}$ We call a group action faithful if no non trivial group element acts by the identity automorphism.
} 
We consider the diagonal action of $\Lambda$ on $A \bar{\otimes} D$ and continue to denote all these actions of $\Lambda$ by $\left(\gamma_{s}\right)_{s \in \Lambda}$.

These data yield the crossed product von Neumann algebra $N=(A \bar{\otimes} D) \rtimes \Lambda$ equipped with the trace Tr induced by $\tau$ and Tr. The action $\left(\alpha_{g}\right)_{g \in \Gamma}$ of $\Gamma$ on $A$ extends to an action on $N$ that equals the identity on $D \rtimes \Lambda$ and that we still denote as $\left(\alpha_{g}\right)_{g \in \Gamma}$. We start by proving a few basic properties.

Lemma 3.1. The von Neumann algebra $N$ is a factor. We have $N \cap(D \rtimes \Lambda)^{\prime}=\mathbb{C} 1$. The action $\left(\alpha_{g}\right)_{g \in \Gamma}$ of $\Gamma$ on $N$ is outer.

Proof. We first prove that $N \cap(D \rtimes \Lambda)^{\prime}=\mathbb{C} 1$. In the case where $D$ is a factor and the action of $\Lambda$ on $D$ is outer, we have that $N \cap(1 \otimes D)^{\prime}=A \otimes 1$. Since the action of $\Lambda$ on $(B, \tau)$ is mixing, the diagonal action on $(A, \tau)$ is still mixing, in particular ergodic, so that $N \cap(D \rtimes \Lambda)^{\prime}=\mathbb{C} 1$.

In the case where $D=L^{\infty}(Z, \eta)$ is diffuse abelian and the action $\Lambda \curvearrowright(Z, \eta)$ is essentially free and ergodic, the essential freeness implies that $N \cap(1 \otimes D)^{\prime}=A \bar{\otimes} D$. So we must prove that all $\Lambda$-invariant elements in $A \bar{\otimes} D$ are scalar multiples of 1 . Let $F: Z \rightarrow A \ominus \mathbb{C} 1$ be a measurable function satisfying $F(s \cdot z)=\gamma_{s}(F(z))$ for all $s \in \Lambda$ and a.e. $z \in Z$. We must prove that $F$ is zero a.e. Since $\Lambda$ acts ergodically on $(Z, \eta)$, the map $z \mapsto\|F(z)\|_{2}$ is constant a.e. If this constant differs from zero, we may assume that it is equal to 1 a.e. We can then choose $a \in A \ominus \mathbb{C} 1$ with $\|a\|_{2}=1$ such that

$$
\mathcal{U}=\left\{z \in Z \mid\|F(z)-a\|_{2}<1 / 3\right\}
$$

is nonnegligible. Since the action of $\Lambda$ on $(A, \tau)$ is mixing, we can take a finite subset $\mathcal{F} \subset \Lambda$ such that $\left|\left\langle\gamma_{s}(a), a\right\rangle\right|<1 / 3$ for all $s \in \Lambda-\mathcal{F}$. We derive as follows that $\eta(s \cdot \mathcal{U} \cap \mathcal{U})=0$ for all $s \in \Lambda-\mathcal{F}$. Indeed, otherwise we find $s \in \Lambda-\mathcal{F}$ and a point $z \in \mathcal{U}$ such that $s \cdot z \in \mathcal{U},\|F(z)\|_{2}=1$ and $\gamma_{s}(F(z))=F(s \cdot z)$. But then we arrive at the contradiction

$$
1=|\langle F(s \cdot z), F(s \cdot z)\rangle|=\left|\left\langle\gamma_{s}(F(z)), F(s \cdot z)\right\rangle\right|<2 / 3+\left|\left\langle\gamma_{s}(a), a\right\rangle\right|<1 .
$$

So for almost every $z \in \mathcal{U}$, we have that $\Lambda \cdot z \cap \mathcal{U} \subset \mathcal{F} \cdot z$. Therefore the restriction of the orbit equivalence relation of $\Lambda \curvearrowright Z$ to the nonnegligible subset $\mathcal{U}$ has finite orbits almost everywhere. But this equivalence relation is ergodic and $\mathcal{U}$ is nonatomic. This is absurd and the conclusion that $N \cap(D \rtimes \Lambda)^{\prime}=\mathbb{C} 1$ follows.

We have in particular that $N$ is a factor. Assume that $g \in \Gamma$ and $V \in \mathcal{U}(N)$ with $\alpha_{g}=\operatorname{Ad} V$. Since $\alpha_{g}(d)=d$ for all $d \in D \rtimes \Lambda$, it follows that $V$ is scalar. Hence $\alpha_{g}=$ id. Since $B \neq \mathbb{C} 1$ and since the action $\left(\beta_{g}\right)_{g \in \Gamma_{0}}$ of $\Gamma_{0}$ on $B$ is faithful, also the action $\left(\alpha_{g}\right)_{g \in \Gamma}$ of $\Gamma$ on $A$ is faithful. We conclude that $g=e$.

We also record the following elementary result that we will need in Section 4. 
Lemma 3.2. Let $\Gamma_{1}<\Gamma$ be a torsion free subgroup and $\Gamma / \Gamma_{0}=I \sqcup J$ a partition of $\Gamma / \Gamma_{0}$ into $\Gamma_{1}$-invariant subsets such that $\Gamma_{1}$ acts freely on I. Define the Hilbert space

$$
L:=L^{2}(N) \ominus L^{2}\left(\left(B^{J} \bar{\otimes} D\right) \rtimes \Lambda\right) .
$$

Then the unitary representation $\left(\alpha_{g}\right)_{g \in \Gamma_{1}}$ of $\Gamma_{1}$ on $L$ is a multiple of the regular representation of $\Gamma_{1}$.

Proof. For every finite nonempty subset $\mathcal{F} \subset I$, define $L_{\mathcal{F}} \subset L$ as the closed linear span of $\left((B \ominus \mathbb{C} 1)^{\mathcal{F}} B^{J} \otimes 1\right) L^{2}(D \rtimes \Lambda)$. Note that $L$ is the orthogonal direct sum of all the $L_{\mathcal{F}}$. Fix a nonempty finite subset $\mathcal{F} \subset I$ and define $\Gamma_{2}=\left\{g \in \Gamma_{1} \mid\right.$ $g \mathcal{F}=\mathcal{F}\}$. Since $\Gamma_{1}$ acts freely on $I$ and since $\mathcal{F}$ is finite, it follows that $\Gamma_{2}$ is a finite subgroup of $\Gamma_{1}$. Since $\Gamma_{1}$ is torsion free, we get that $\Gamma_{2}=\{e\}$. Because $\alpha_{g}\left(L_{\mathcal{F}}\right)=L_{g \mathcal{F}}$, we conclude that the subspaces $\left(\alpha_{g}\left(L_{\mathcal{F}}\right)\right)_{g \in \Gamma_{1}}$ are orthogonal and the lemma is proved.

The aim of this section is to understand when these actions $\left(\alpha_{g}\right)_{g \in \Gamma}$ are outer conjugate, keeping fixed $\Gamma_{0}<\Gamma$ but varying all the other data.

So we keep $\Gamma_{0}<\Gamma$ fixed, but further assume that we have, for $i=1,2$, von Neumann algebras $\left(B_{i}, \tau\right)$ and $\left(D_{i}, \operatorname{Tr}\right)$, infinite groups $\Lambda_{i}$ and actions $\left(\beta_{g}^{i}\right)_{g \in \Gamma_{0}}$ and $\left(\gamma_{s}^{i}\right)_{s \in \Lambda_{i}}$. This results into factors $N_{i}$ with outer actions $\left(\alpha_{g}^{i}\right)_{g \in \Gamma}$.

Throughout, we keep as standing assumptions the properties listed in the beginning of this section.

Lemma 3.3. Assume that $\psi: N_{1} \rightarrow N_{2}$ is an outer conjugacy between $\left(\alpha_{g}^{1}\right)_{g \in \Gamma}$ and

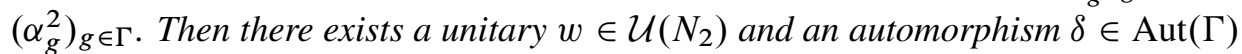
such that the isomorphism $\psi^{\prime}=(\operatorname{Ad} w) \circ \psi$ satisfies

$$
\begin{aligned}
& \psi^{\prime}\left(D_{1} \rtimes \Lambda_{1}\right)=D_{2} \rtimes \Lambda_{2}, \quad \psi^{\prime}\left(D_{1}\right)=D_{2} \quad \text { and } \quad \psi^{\prime} \circ \alpha_{g}^{1}=\alpha_{\delta(g)}^{2} \circ \psi^{\prime} \\
& \text { for all } g \in \Gamma \text {. }
\end{aligned}
$$

Our proof of Lemma 3.3 is very similar to the proof of [29, Theorem 4.1]. We use the spectral gap methods of [29] and the malleable deformation for co-induced actions developed in $[26,28]$. We more precisely use the following variant of that malleable deformation, due to [15]. To introduce the notations, we drop the indices $i=1,2$ from $B_{i}, A_{i}, D_{i}$, etc.

Define $\widetilde{B}=B * L \mathbb{Z}$ with respect to the natural tracial states that we all denote by $\tau$. Denote by $\left(u_{n}\right)_{n \in \mathbb{Z}}$ the canonical unitaries in $L \mathbb{Z}$ and define $h \in L \mathbb{Z}$ as the selfadjoint element with spectrum $[-\pi, \pi]$ satisfying $u_{1}=\exp (i h)$. For every $t \in \mathbb{R}$, we put $u_{t}=\exp (i t h)$ and we define the 1-parameter group $\left(\zeta_{t}\right)_{t \in \mathbb{R}}$ of inner automorphisms of $\widetilde{B}$ given by $\zeta_{t}=\operatorname{Ad} u_{t}$. We extend the actions $\left(\beta_{g}\right)_{g \in \Gamma_{0}}$ and $\left(\gamma_{s}\right)_{s \in \Lambda}$ to $\widetilde{B}$ by acting trivially on $L \mathbb{Z}$. These two actions and the action $\left(\zeta_{t}\right)_{t \in \mathbb{R}}$ all commute. 
Define $\widetilde{A}$ as the infinite tensor product $\widetilde{A}=\widetilde{B}^{\Gamma / \Gamma_{0}}$. We continue to denote by $\left(\gamma_{s}\right)_{s \in \Lambda}$ and $\left(\zeta_{t}\right)_{t \in \mathbb{R}}$ the diagonal actions on $\widetilde{A}$. They commute with the co-induced action $\left(\alpha_{g}\right)_{g \in \Gamma}$ on $\widetilde{A}$. Moreover this co-induced action extends the action $\left(\alpha_{g}\right)_{g \in \Gamma}$ on $A$. We finally consider the crossed product $\widetilde{N}=(\widetilde{A} \bar{\otimes} D) \rtimes \Lambda$ with respect to the diagonal action of $\Lambda$, together with the action $\left(\alpha_{g}\right)_{g \in \Gamma}$ of $\Gamma$ on $\widetilde{N}$ that extends the given action on $\widetilde{A}$ and that is the identity on $D \rtimes \Lambda$.

Our assumption that $\Gamma_{0}$ is not co-amenable in $\Gamma$ is used to obtain the following result.

Lemma 3.4. Assume that $\left(V_{g}\right)_{g \in \Gamma}$ are unitaries in $\mathcal{U}(N)$ and that $\Omega: \Gamma \times \Gamma \rightarrow \mathbb{T}$ is a map satisfying $V_{g} \alpha_{g}\left(V_{h}\right)=\Omega(g, h) V_{g h}$ for all $g, h \in \Gamma$. The unitary representation

$$
\rho: \Gamma \rightarrow \mathcal{U}\left(L^{2}(\widetilde{N} \ominus N)\right): \rho_{g}(\xi)=V_{g} \alpha_{g}(\xi) V_{g}^{*}
$$

does not weakly contain the trivial representation.

Proof. We write $H=L^{2}(\widetilde{N} \ominus N)$. Denote by $\mathcal{S}$ the set of all finite nonempty subsets $\mathcal{F} \subset \Gamma / \Gamma_{0}$. For every $\mathcal{F} \in \mathcal{S}$, we define $H(\mathcal{F})$ as the closed linear span of $\left((\widetilde{B} \ominus B)^{\mathcal{F}} \otimes 1\right) L^{2}(N)$ inside $L^{2}(\widetilde{N} \ominus N)$. One checks that $H$ is the orthogonal direct sum of the subspaces $H(\mathcal{F}), \mathcal{F} \in \mathcal{S}$. Note that $H(\mathcal{F})$ is an $N-N$-subbimodule of $L^{2}(\widetilde{N} \ominus N)$ and that $\rho_{g}(H(\mathcal{F}))=H(g \mathcal{F})$ for all $g \in \Gamma$ and $\mathcal{F} \in \mathcal{S}$.

Denote by $\operatorname{Prob}(\mathcal{S})$ the set of probability measures on the countable set $\mathcal{S}$. Denote by $(H)_{1}$ the set of unit vectors in $H$. The map

$$
\theta:(H)_{1} \rightarrow \operatorname{Prob}(\mathcal{S}):(\theta(\xi))(\mathcal{F})=\left\|P_{H(\mathcal{F})}(\xi)\right\|_{2}^{2}
$$

satisfies $\theta\left(\rho_{g}(\xi)\right)=g \cdot \theta(\xi)$.

Assume now that the unitary representation $\rho$ weakly contains the trivial representation, i.e. admits a sequence of unit vectors $\xi_{n} \in(H)_{1}$ satisfying $\lim _{n}\left\|\rho_{g}\left(\xi_{n}\right)-\xi_{n}\right\|_{2}=0$ for all $g \in \Gamma$. We will prove that $\Gamma_{0}$ is co-amenable in $\Gamma$. Define $\omega_{n}=\theta\left(\xi_{n}\right)$. Then $\omega_{n}$ is a sequence of probability measures on $\mathcal{S}$ satisfying $\lim _{n}\left\|g \cdot \omega_{n}-\omega_{n}\right\|_{1}=0$.

Choose a set $\mathcal{S}_{0} \subset \mathcal{S}$ of representatives for the orbits of the action $\Gamma \curvearrowright \mathcal{S}$. We make this choice such that $e \Gamma_{0} \in \mathcal{F}$ for every $\mathcal{F} \in \mathcal{S}_{0}$. For every $\mathcal{F} \in \mathcal{S}_{0}$, define $\operatorname{Norm}(\mathcal{F})=\{g \in \Gamma \mid g \mathcal{F}=\mathcal{F}\}$. Since $\mathcal{S}_{0}$ is a set of representatives for the action $\Gamma \curvearrowright \mathcal{S}$, we identify $\mathcal{S}$ with the disjoint union of the sets $\Gamma / \operatorname{Norm}(\mathcal{F}), \mathcal{F} \in \mathcal{S}_{0}$.

For every $\mathcal{F} \in \mathcal{S}_{0}$, write $\operatorname{Stab}(\mathcal{F})=\left\{g \in \Gamma \mid g h \Gamma_{0}=h \Gamma_{0}\right.$ for all $\left.h \Gamma_{0} \in \mathcal{F}\right\}$. Since all the $\mathcal{F}$ are finite sets, we have that $\operatorname{Stab}(\mathcal{F})$ is a finite index subgroup of $\operatorname{Norm}(\mathcal{F})$. We define $\mathcal{S}^{\prime}$ as the disjoint union of the sets $\Gamma / \operatorname{Stab}(\mathcal{F}), \mathcal{F} \in \mathcal{S}_{0}$. Putting together the finite-to-one maps $\Gamma / \operatorname{Stab}(\mathcal{F}) \rightarrow \Gamma / \operatorname{Norm}(\mathcal{F})$, we obtain the $\Gamma$-equivariant finite-to-one map $\theta^{\prime}: \mathcal{S}^{\prime} \rightarrow \mathcal{S}$. This map $\theta^{\prime}$ induces a $\Gamma$-equivariant isometry of $\operatorname{Prob}(\mathcal{S})$ into $\operatorname{Prob}\left(\mathcal{S}^{\prime}\right)$. Applying this isometry to $\omega_{n}$, we find a sequence of probability measures $\omega_{n}^{\prime}$ on $\mathcal{S}^{\prime}$ satisfying $\lim _{n}\left\|g \cdot \omega_{n}^{\prime}-\omega_{n}^{\prime}\right\|_{1}=0$ for all $g \in \Gamma$. 
Taking a weak* limit point, it follows that the action $\Gamma \curvearrowright \mathcal{S}^{\prime}$ admits an invariant mean. For every $\mathcal{F} \in \mathcal{S}_{0}$, we have $e \Gamma_{0} \in \mathcal{F}$ and therefore $\operatorname{Stab}(\mathcal{F}) \subset \Gamma_{0}$. Define the map $\theta^{\prime \prime}: \mathcal{S}^{\prime} \rightarrow \Gamma / \Gamma_{0}$ given by $\theta^{\prime \prime}(h \operatorname{Stab}(\mathcal{F}))=h \Gamma_{0}$ for all $\mathcal{F} \in \mathcal{S}_{0}$ and $h \in \Gamma$. Since $\theta^{\prime \prime}$ is $\Gamma$-equivariant, we push forward the $\Gamma$-invariant mean on $\mathcal{S}^{\prime}$ to a $\Gamma$ invariant mean on $\Gamma / \Gamma_{0}$. This precisely means that $\Gamma_{0}$ is co-amenable inside $\Gamma$.

We are now ready to prove Lemma 3.3.

Proof of Lemma 3.3. Fix a projection $p_{1} \in D_{1} \subset N_{1}$ with $0<\operatorname{Tr}\left(p_{1}\right)<\infty$. After unitarily conjugating $\psi$, we may assume that $\psi\left(p_{1}\right) \in D_{2}$. Put $p_{2}=\psi\left(p_{1}\right)$.

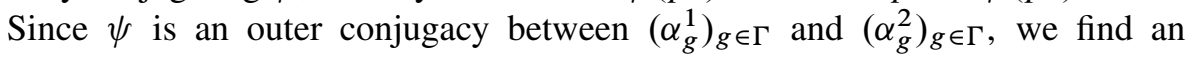
automorphism $\delta \in \operatorname{Aut}(\Gamma)$, unitaries $\left(V_{g}\right)_{g \in \Gamma}$ in $\mathcal{U}\left(N_{2}\right)$ and a map $\Omega: \Gamma \times \Gamma \rightarrow \mathbb{T}$ such that

$\psi \circ \alpha_{\delta^{-1}(g)}^{1}=\left(\operatorname{Ad} V_{g}\right) \circ \alpha_{g}^{2} \circ \psi \quad$ and $\quad V_{g} \alpha_{g}^{2}\left(V_{h}\right)=\Omega(g, h) V_{g h} \quad$ for all $g, h \in \Gamma$.

Define as above the malleable deformation $\left(\zeta_{t}\right)_{t \in \mathbb{R}}$ of $\widetilde{N}_{2}=\left(\widetilde{A}_{2} \bar{\otimes} D_{2}\right) \rtimes \Lambda_{2}$. Denote by $\rho$ the unitary representation of $\Gamma$ on $L^{2}\left(\widetilde{N}_{2} \ominus N_{2}\right)$ given by $\rho_{g}(\xi)=V_{g} \alpha_{g}^{2}(\xi) V_{g}^{*}$. By Lemma 3.4, $\rho$ does not weakly contain the trivial representation. We then find a constant $\kappa>0$ and a finite subset $\mathcal{F} \subset \Gamma$ such that

$$
\|\xi\|_{2} \leq \kappa \sum_{g \in \mathcal{F}}\left\|\rho_{g}(\xi)-\xi\right\|_{2} \quad \text { for all } \xi \in L^{2}\left(\widetilde{N}_{2} \ominus N_{2}\right) .
$$

We write $M_{i}=p_{i} N_{i} p_{i}$ and $\widetilde{M}_{i}=p_{i} \widetilde{N}_{i} p_{i}$. Put $P_{i}=p_{i}\left(D_{i} \rtimes \Lambda_{i}\right) p_{i}$. Put $\varepsilon=\left\|p_{2}\right\|_{2} / 4$ and $\delta=\varepsilon /(2 \kappa|\mathcal{F}|)$. Take an integer $n_{0}$ large enough such that $t=n_{0}^{-1}$ satisfies

$$
\left\|\left(V_{g}-\zeta_{t}\left(V_{g}\right)\right) p_{2}\right\|_{2} \leq \delta \text { for all } g \in \mathcal{F} .
$$

For every $a \in P_{1}$ and $g \in \Gamma$, we have $\alpha_{g}^{1}(a)=a$ and therefore $V_{g} \alpha_{g}^{2}(\psi(a)) V_{g}^{*}=$ $\psi(a)$. By our choice of $t$, we get that

$\left\|V_{g} \alpha_{g}^{2}\left(\zeta_{t}(b)\right) V_{g}^{*}-\zeta_{t}(b)\right\|_{2} \leq 2 \delta \quad$ for all $g \in \mathcal{F}$ and all $b \in \psi\left(P_{1}\right)$ with $\|b\| \leq 1$.

Denote by $E: \widetilde{N} \rightarrow N$ the unique trace preserving conditional expectation. Whenever $b \in \psi\left(P_{1}\right)$ with $\|b\| \leq 1$, we put $\xi=\zeta_{t}(b)-E\left(\zeta_{t}(b)\right)$ and conclude from (3.2) that

$$
\kappa \sum_{g \in \mathcal{F}}\left\|\rho_{g}(\xi)-\xi\right\|_{2} \leq 2 \kappa|\mathcal{F}| \delta=\varepsilon .
$$

It follows from (3.1) that $\|\xi\|_{2} \leq \varepsilon$. A direct computation shows that $\left(\zeta_{t}\right)$ satisfies the following transversality property of [29, Lemma 2.1].

$$
\left\|b-\zeta_{t}(b)\right\|_{2} \leq \sqrt{2}\left\|\zeta_{t}(b)-E\left(\zeta_{t}(b)\right)\right\|_{2} \text { for all } b \in M_{2} .
$$


We conclude that $\left\|b-\zeta_{t}(b)\right\|_{2} \leq 2 \varepsilon$ for all $b \in \psi\left(P_{1}\right)$ with $\|b\| \leq 1$. It follows that for all $b \in \mathcal{U}\left(\psi\left(P_{1}\right)\right)$,

$$
\left|\operatorname{Tr}\left(b \zeta_{t}\left(b^{*}\right)\right)-\operatorname{Tr}\left(b b^{*}\right)\right| \leq\|b\|_{2}\left\|b-\zeta_{t}(b)\right\|_{2} \leq\left\|p_{2}\right\|_{2} 2 \varepsilon=\operatorname{Tr}\left(p_{2}\right) / 2 .
$$

So $\operatorname{Tr}\left(b \zeta_{t}\left(b^{*}\right)\right) \geq \operatorname{Tr}\left(p_{2}\right) / 2$ for all $b \in \mathcal{U}\left(\psi\left(P_{1}\right)\right)$.

Defining $W \in \widetilde{M}_{2}$ as the unique element of minimal $\|\cdot\|_{2}$ in the weakly closed convex hull of $\left\{b \zeta_{t}\left(b^{*}\right) \mid b \in \mathcal{U}\left(\psi\left(P_{1}\right)\right)\right\}$, it follows that $\operatorname{Tr}(W) \geq \operatorname{Tr}\left(p_{2}\right) / 2$ and $b W=W \zeta_{t}(b)$ for all $b \in \psi\left(P_{1}\right)$. In particular, $W$ is a nonzero element of $\widetilde{M}_{2}$ and $W W^{*}$ commutes with $\psi\left(P_{1}\right)$.

Since $V_{g} \alpha_{g}^{2}(b) V_{g}^{*}=b$ for all $g \in \Gamma$ and all $b \in \psi\left(P_{1}\right)$, the elements $W_{g}:=V_{g} \alpha_{g}^{2}(W) \zeta_{t}\left(V_{g}^{*}\right)$ also satisfy $b W_{g}=W_{g} \zeta_{t}(b)$ for all $b \in \psi\left(P_{1}\right)$. The join of the left support projections of all $W_{g}, g \in \Gamma$, is a projection $q \in \widetilde{M}_{2} \cap \psi\left(P_{1}\right)^{\prime}$ that satisfies $q=V_{g} \alpha_{g}^{2}(q) V_{g}^{*}$ for all $g \in \Gamma$. By Lemma 3.4, $q \in M_{2}$. But then, by Lemma 3.1, we get that $q \in \psi\left(M_{1} \cap P_{1}^{\prime}\right)=\mathbb{C} p_{2}$. Since $q$ is nonzero, we conclude that $q=p_{2}$. It follows that we can find a $g \in \Gamma$ such that $W^{\prime}=W \zeta_{t}\left(W_{g}\right)$ is nonzero. By construction, we have $b W^{\prime}=W^{\prime} \zeta_{2 t}(b)$ for all $b \in \psi\left(P_{1}\right)$. We can repeat the same reasoning inductively. Since $t=1 / n_{0}$, we find a nonzero element $W \in \widetilde{M}_{2}$ satisfying $b W=W \zeta_{1}(b)$ for all $b \in \psi\left(P_{1}\right)$.

For every finite subset $\mathcal{F} \subset \Gamma / \Gamma_{0}$, we define $M_{2}(\mathcal{F})=p_{2}\left(\left(B_{2}^{\mathcal{F}} \bar{\otimes} D_{2}\right) \rtimes \Lambda_{2}\right) p_{2}$. We claim that there exists a finite subset $\mathcal{F} \subset \Gamma / \Gamma_{0}$ such that $\psi\left(P_{1}\right) \prec_{2} M_{2}(\mathcal{F})$. Indeed, if this is not the case, we find a sequence of unitaries $b_{n} \in \mathcal{U}\left(\psi\left(P_{1}\right)\right)$ satisfying

$$
\left\|E_{M_{2}(\mathcal{F})}\left(x b_{n} y\right)\right\|_{2} \rightarrow 0 \text { for all } x, y \in M_{2} \text { and all finite subsets } \mathcal{F} \subset \Gamma / \Gamma_{0} .
$$

We claim that

$$
\left\|E_{M_{2}}\left(x \zeta_{1}\left(b_{n}\right) y\right)\right\|_{2} \rightarrow 0 \text { for all } x, y \in \widetilde{M}_{2} \text {. }
$$

Since the linear span of all $M_{2} \widetilde{B}_{2}^{\mathcal{F}}, \mathcal{F} \subset \Gamma / \Gamma_{0}$ finite, is $\|\cdot\|_{2}$-dense in $\widetilde{M}_{2}$, it suffices to prove (3.3) for all $x, y \in \widetilde{B}_{2}^{\mathcal{F}} p_{2}$ and all finite subsets $\mathcal{F} \subset \Gamma / \Gamma_{0}$. But for such $x, y \in \widetilde{B}_{2}^{\mathcal{F}} p_{2}$, we have

$$
E_{M_{2}}\left(x \zeta_{1}\left(b_{n}\right) y\right)=E_{M_{2}}\left(x \zeta_{1}\left(E_{M_{2}(\mathcal{F})}\left(b_{n}\right)\right) y\right)
$$

and the conclusion follows from our choice of $\left(b_{n}\right)$. So (3.3) is proven. It follows in particular that $\left\|E_{M_{2}}\left(W \zeta_{1}\left(b_{n}\right) W^{*}\right)\right\|_{2} \rightarrow 0$. Since

$$
E_{M_{2}}\left(W \zeta_{1}\left(b_{n}\right) W^{*}\right)=E_{M_{2}}\left(b_{n} W W^{*}\right)=b_{n} E_{M_{2}}\left(W W^{*}\right)
$$

and since $b_{n}$ is unitary, we conclude that $W W^{*}=0$. This is absurd and we have proven the existence of a finite subset $\mathcal{F} \subset \Gamma / \Gamma_{0}$ such that $\psi\left(P_{1}\right) \prec_{M_{2}} M_{2}(\mathcal{F})$. 
Note that $\psi\left(P_{1}\right) \nprec p_{2}\left(B_{2}^{\mathcal{F}} \bar{\otimes} D_{2}\right) p_{2}$, because otherwise we can take the relative commutant, apply [36, Lemma 3.5] and reach the contradiction that

$$
\left(B_{2}^{\Gamma / \Gamma_{0}-\mathcal{F}} \otimes 1\right) p_{2} \prec M_{2} \cap \psi\left(P_{1}\right)^{\prime}=\psi\left(M_{1} \cap P_{1}^{\prime}\right)=\mathbb{C} p_{2} .
$$

In combination with the previous paragraph and [36, Remark 3.8], we find projections $q_{1} \in P_{1}$ and $q_{2} \in p_{2} D_{2} p_{2}$, a $*$-homomorphism $\theta: q_{1} P_{1} q_{1} \rightarrow q_{2} M_{2}(\mathcal{F}) q_{2}$ and a nonzero partial isometry $V \in \psi\left(q_{1}\right) M_{2} q_{2}$ satisfying $\psi(b) V=V \theta(b)$ for all $b \in q_{1} P_{1} q_{1}$, and satisfying $\theta\left(q_{1} P_{1} q_{1}\right) \nprec q_{2}\left(B_{2}^{\mathcal{F}} \bar{\otimes} D_{2}\right) q_{2}$.

The projection $V V^{*}$ commutes with $\psi\left(q_{1} P_{1} q_{1}\right)$ and hence must be equal to $\psi\left(q_{1}\right)$. The projection $V^{*} V$ commutes with $\theta\left(q_{1} P_{1} q_{1}\right)$. Since the action of $\Lambda_{2}$ on $B_{2}^{\Gamma / \Gamma_{0}-\mathcal{F}}$ is mixing, it follows from Lemma 2.1 that $V^{*} V \in q_{2} M_{2}(\mathcal{F}) q_{2}$. So we may assume that $V^{*} V=q_{2}$. Since $P_{1}$ and $M_{2}(\mathcal{F})$ are factors, we can then amplify $V$ to a unitary element $V \in \mathcal{U}\left(M_{2}\right)$ satisfying $V^{*} \psi\left(P_{1}\right) V \subset M_{2}(\mathcal{F})$.

Since $\Gamma_{0}$ is not co-amenable inside $\Gamma$, it certainly has infinite index. Therefore we can find $g \in \Gamma$ such that $g \mathcal{F} \cap \mathcal{F}=\emptyset$ (see e.g. [30, Lemma 2.4]). Denote $Q_{2}=V^{*} \psi\left(P_{1}\right) V$. So $Q_{2} \subset M_{2}(\mathcal{F})$. Since $\alpha_{g}^{1}\left(P_{1}\right)=P_{1}$, it follows that the von Neumann algebras $Q_{2}$ and $\alpha_{g}^{2}\left(Q_{2}\right)$ are unitarily conjugate inside $M_{2}$. Since $Q_{2} \subset M_{2}(\mathcal{F})$ and $\alpha_{g}^{2}\left(Q_{2}\right) \subset M_{2}(g \mathcal{F})$, it follows from Lemma 2.2 that $Q_{2} \prec M_{2}(\emptyset)=P_{2}$. Reasoning as above, we find a unitary $V \in \mathcal{U}\left(M_{2}\right)$ such that $V^{*} \psi\left(P_{1}\right) V \subset P_{2}$.

The same reasoning applies to $\psi^{-1}$ and we also find $W \in \mathcal{U}\left(M_{1}\right)$ such that $W^{*} \psi^{-1}\left(P_{2}\right) W \subset P_{1}$. Writing $T=\psi(W) V$, we get that

$$
T^{*} P_{2} T \subset V^{*} \psi\left(P_{1}\right) V \subset P_{2} .
$$

Since the action of $\Lambda_{2}$ on $A_{2}$ is mixing, it follows from Lemma 2.1 that $T \in P_{2}$. But then the inclusions in (3.4) are equalities and we conclude that $V^{*} \psi\left(P_{1}\right) V=P_{2}$.

So after a unitary conjugacy of $\psi$, we may from now on assume that $\psi\left(D_{1} \rtimes \Lambda_{1}\right)$ $=D_{2} \rtimes \Lambda_{2}$ and $\psi\left(p_{1}\right)=p_{2}$. Inside $P_{2}$, we must have the embedding $\psi\left(p_{1} D_{1} p_{1}\right) \prec p_{2} D_{2} p_{2}$. Indeed, since the action $\Lambda_{2} \curvearrowright A_{2}$ is mixing and $\psi\left(A_{1} p_{1}\right)$ commutes with $\psi\left(p_{1} D_{1} p_{1}\right)$, it would otherwise follow from Lemma 2.1 that $\psi\left(A_{1} p_{1}\right) \subset P_{2}$ and hence $\psi\left(M_{1}\right) \subset P_{2}$, which is absurd. We have a similar embedding statement for $\psi^{-1}$.

In the case where the $D_{i}$ are abelian, $D_{i} p_{i}$ is a Cartan subalgebra of $P_{i}$. It then follows from [27, Theorem A.1] that $\psi\left(D_{1} p_{1}\right)$ can be unitarily conjugated onto $D_{2} p_{2}$ inside $P_{2}$. In the case where the $D_{i}$ are factors and the groups $\Lambda_{i}$ have a torsion free FC-radical, [17, Lemma 8.4] yields the same conclusion ${ }^{4}$. So

${ }^{4}$ The statement of [17, Lemma 8.4] requires the groups $\Lambda_{i}$ to be icc, but the proof of [17, Lemma 8.4] only uses the following property : if $K<\Lambda_{i}$ is a finite subgroup and $H<\Lambda_{i}$ is a finite index subgroup such that $K$ is normal in $H$, then $K=\{e\}$. This last property is equivalent with the torsion freeness of the FC-radical of $\Lambda_{i}$. 
after a further unitary conjugacy of $\psi$, with a unitary from $D_{2} \rtimes \Lambda_{2}$, we arrive at $\psi\left(D_{1} \rtimes \Lambda_{1}\right)=D_{2} \rtimes \Lambda_{2}$ and $\psi\left(D_{1}\right)=D_{2}$.

Using Lemma 3.1, we then get that $V_{g} \in N_{2} \cap\left(D_{2} \rtimes \Lambda_{2}\right)^{\prime}=\mathbb{C} 1$ and hence, $\psi \circ \alpha_{g}^{1}=\alpha_{\delta}^{2}(g) \circ \psi$ for all $g \in \Gamma$.

Theorem B is an immediate consequence of Lemma 3.3.

Proof of Theorem B. Assume that $\alpha^{\Lambda_{1}}$ and $\alpha^{\Lambda_{2}}$ are outer conjugate. Then Lemma 3.3 yields an isomorphism

$$
\psi: M_{2}(\mathbb{C})^{\Lambda_{1}} \rtimes \Lambda_{1} \rightarrow M_{2}(\mathbb{C})^{\Lambda_{2}} \rtimes \Lambda_{2} \quad \text { with } \quad \psi\left(M_{2}(\mathbb{C})^{\Lambda_{1}}\right)=M_{2}(\mathbb{C})^{\Lambda_{2}}
$$

It follows that $\Lambda_{1} \cong \Lambda_{2}$. The converse is obvious.

\section{Proof of Theorem A}

Theorem A will be derived as a consequence of the more general Theorem 4.2 below.

Assumptions 4.1. We use, as a black box, the following kind of measure preserving automorphism $T$ of a standard nonatomic probability space $(Y, v)$.

(1) $T$ is mixing.

(2) The only automorphisms of $(Y, v)$ that commute with $T$ are the powers of $T$.

(3) The automorphisms $T$ and $T^{-1}$ are not isomorphic: there is no $S \in \operatorname{Aut}(Y, v)$ satisfying $S T S^{-1}=T^{-1}$.

(4) Viewing $T$ as a unitary operator on $L^{2}(Y, v)$, its maximal spectral type is singular w.r.t. the Lebesgue measure.

In [34, Theorems 2.7 and 2.8], it was shown that Ornstein's rank one automorphisms of [21] satisfy conditions 1, 2 and 3. In [5], these automorphisms were proven to satisfy condition 4 as well. Note that in [34], conditions 2 and 3 are deduced from a stronger property of $T$ : the mixing automorphism $T$ actually has minimal self joinings (MSJ) in the strongest possible sense saying that the only measures on $Y \times Y$ that are invariant under $T^{n} \times T^{m}$ (with $n \neq 0$ and $m \neq 0$ ) and that have marginals $v$, are the obvious ones. In later articles, the notion of MSJ has been weakened by only considering $T \times T$ invariant measures with marginals $v$. We refer to [8, Proposition 6.7] for a detailed discussion.

Every automorphism $T \in \operatorname{Aut}(Y, v)$ satisfying the assumptions in 4.1 gives rise to a mixing pmp action $\mathbb{Z} \curvearrowright(Y, \nu)$ that we denote by $\left(\gamma_{n}\right)_{n \in \mathbb{Z}}$ and that has the following properties : the normalizer of $\mathbb{Z}$ inside $\operatorname{Aut}(Y, v)$ equals $\mathbb{Z}$ itself; and there is no nonzero bounded operator $L^{2}(Y, v) \rightarrow \ell^{2}(\mathbb{Z})$ that intertwines the unitary representation $\mathbb{Z} \curvearrowright L^{2}(Y, v)$ induced by $\left(\gamma_{n}\right)_{n \in \mathbb{Z}}$ with the regular representation of $\mathbb{Z}$. 
Theorem 4.2. Let $\Gamma$ be any fixed nonamenable group that contains a copy of $\mathbb{Z}$ as a malnormal ${ }^{5}$ subgroup $\Gamma_{0}$. Fix an automorphism $T \in \operatorname{Aut}(Y, v)$ satisfying the assumptions in 4.1 and consider the associated action of $\Gamma_{0}=\mathbb{Z}$ on $(Y, v)$. Put $(X, \mu)=(Y, v)^{\Gamma / \Gamma_{0}}$ and consider the coinduced action $\Gamma \curvearrowright(X, \mu)$ as well as the diagonal action $\mathbb{Z} \curvearrowright(X, \mu)$. Fix a standard nonatomic finite or infinite measure space $(Z, \eta)$.

Whenever $\Delta \in \operatorname{Aut}(Z, \eta)$ is ergodic and measure preserving, consider

$$
N=L^{\infty}(X \times Z) \rtimes \mathbb{Z}
$$

where $\mathbb{Z}$ acts diagonally on $X \times Z$. Consider the action $\left(\alpha_{g}^{\Delta}\right)_{g \in \Gamma}$ of $\Gamma$ on $N$ that extends the action $\Gamma \curvearrowright(X, \mu)$ and that is the identity on $L^{\infty}(Z) \rtimes \mathbb{Z}$.

If $\Delta_{1}, \Delta_{2} \in \operatorname{Aut}(Z, \eta)$ are ergodic and measure preserving and if $\psi: N_{1} \rightarrow N_{2}$ is an outer conjugacy between $\left(\alpha_{g}^{\Delta_{1}}\right)_{g \in \Gamma}$ and $\left(\alpha_{g}^{\Delta_{2}}\right)_{g \in \Gamma \text {, there exists a unitary }}$ $w \in \mathcal{U}\left(N_{2}\right)$ and a group element $g_{0} \in \Gamma$ such that the outer conjugacy $\psi^{\prime}=\alpha_{g_{0}}^{2} \circ(\operatorname{Ad} w) \circ \psi$ is the composition of

- the isomorphism $N_{1} \rightarrow N_{2}$ induced by an automorphism $\theta \in \operatorname{Aut}(Z, \eta)$ satisfying $\theta \circ \Delta_{1} \circ \theta^{-1}=\Delta_{2}$; this isomorphism acts as the identity on $L^{\infty}(X) \rtimes \mathbb{Z}$ and as $\theta_{*}$ on $L^{\infty}(Z)$;

- the automorphism of $N_{2}$ induced by an automorphism $\delta \in \operatorname{Aut}(\Gamma)$ satisfying $\delta(g)=g$ for all $g \in \Gamma_{0}$; this automorphism acts as the identity on $L^{\infty}(Z) \rtimes \mathbb{Z}$ and acts by permuting the tensor factors of $L^{\infty}(X, \mu)=$ $L^{\infty}(Y, v)^{\Gamma / \Gamma_{0}}$ by the permutation $g \Gamma_{0} \mapsto \delta(g) \Gamma_{0}, g \in \Gamma$.

We say that actions $\left(\alpha_{g}^{i}\right)_{g \in \Gamma}, i=1,2$, of a group $\Gamma$ on von Neumann algebras $N_{i}$ are isomorphic if there exists an isomorphism $\psi: N_{1} \rightarrow N_{2}$ such that $\alpha_{g}^{2} \circ \psi=\psi \circ \alpha_{g}^{1}$ for all $g \in \Gamma$. We also use the notations $\bmod (\psi)$ and $\bmod (\theta)$ to denote the scaling factor of a trace scaling automorphism $\psi \in \operatorname{Aut}(N)$, or a measure scaling automorphism $\theta \in \operatorname{Aut}(Z, \eta)$.

As a consequence of Theorem 4.2, we then have the following results.

$$
\begin{aligned}
\{\bmod (\psi) \mid \psi \in \operatorname{Aut}(N) & \text { is an outer conjugacy of } \left.\alpha^{\Delta}\right\} \\
& =\left\{\bmod (\psi) \mid \psi \in \operatorname{Aut}(N) \text { commutes with } \alpha^{\Delta}\right\} \\
& =\{\bmod (\theta) \mid \theta \in \operatorname{Aut}(Z, \eta) \text { commutes with } \Delta\} .
\end{aligned}
$$

If $\Delta_{1}, \Delta_{2} \in \operatorname{Aut}(Z, \eta)$ are ergodic and measure preserving, it follows from Theorem 4.2 that the following three statements are equivalent.

- $\Delta_{1}$ is conjugate with $\Delta_{2}$ : there exists a nonsingular automorphism $\theta \in \operatorname{Aut}(Z, \eta)$ such that $\Delta_{2}=\theta \circ \Delta_{1} \circ \theta^{-1}$ a.e.

- The actions $\left(\alpha_{g}^{\Delta_{1}}\right)_{g \in \Gamma}$ and $\left(\alpha_{g}^{\Delta_{2}}\right)_{g \in \Gamma}$ are isomorphic.

- The actions $\left(\alpha_{g}^{\Delta_{1}}\right)_{g \in \Gamma}$ and $\left(\alpha_{g}^{\Delta_{2}}\right)_{g \in \Gamma}$ are outer conjugate.

\footnotetext{
${ }^{5}$ A subgroup $\Gamma_{0}<\Gamma$ is said to be malnormal if $g \Gamma_{0} g^{-1} \cap \Gamma_{0}=\{e\}$ for all $g \in \Gamma-\Gamma_{0}$.
} 
Proof of Theorem 4.2. We start by making the notations compatible with those at the beginning of Section 3. We denote $B=L^{\infty}(Y, v)$ and $A=B^{\Gamma / \Gamma_{0}}=L^{\infty}(X, \mu)$. For every $g \Gamma_{0} \in \Gamma / \Gamma_{0}$, we denote by $\pi_{g \Gamma_{0}}: B \rightarrow A$ the embedding of $B$ as the $g \Gamma_{0}$-th tensor factor of $A=B^{\Gamma / \Gamma_{0}}$. We have $\Gamma_{0}=\mathbb{Z}=\Lambda$ and the actions $\left(\beta_{g}\right)_{g \in \Gamma_{0}}$ and $\left(\gamma_{g}\right)_{g \in \Gamma_{0}}$ on $B$ are equal and both induced by $T$.

We write $D=L^{\infty}(Z, \eta)$. The ergodic measure preserving automorphisms $\Delta_{1}, \Delta_{2} \in \operatorname{Aut}(Z, \eta)$ induce essentially free and ergodic actions $\left(\gamma_{n}^{i}\right)_{n \in \mathbb{Z}}$ of $\Lambda=\mathbb{Z}$ on $D$. We consider $N_{1}, N_{2}$ as in the formulation of the Theorem, but we denote the

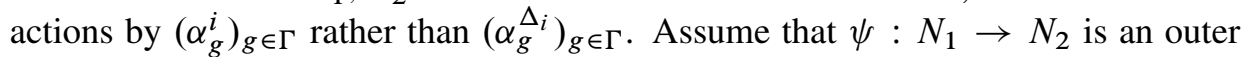
conjugacy between $\left(\alpha_{g}^{1}\right)_{g \in \Gamma}$ and $\left(\alpha_{g}^{2}\right)_{g \in \Gamma}$.

By Lemma 3.3, and after replacing $\psi$ by $(\operatorname{Ad} w) \circ \psi$, we may assume that

$$
\psi\left(D \rtimes_{\gamma^{1}} \mathbb{Z}\right)=D \rtimes_{\gamma^{2}} \mathbb{Z} \quad, \quad \psi(D)=D \quad \text { and } \quad \psi \circ \alpha_{g}^{1}=\alpha_{\delta(g)}^{2} \circ \psi
$$

for all $g \in \Gamma$ and some automorphism $\delta \in \operatorname{Aut}(\Gamma)$. Taking the relative commutant of $\psi(D)=D$, we also have that $\psi(A \bar{\otimes} D)=A \bar{\otimes} D$.

We prove now the existence of a $g_{0} \in \Gamma$ such that $g_{0} \delta\left(\Gamma_{0}\right) g_{0}^{-1} \cap \Gamma_{0} \neq\{e\}$. If such a $g_{0}$ does not exist, it follows from Lemma 3.2 that the unitary representation $\left(\alpha_{\delta(g)}^{2}\right)_{g \in \Gamma_{0}}$ on $L^{2}\left(N_{2}\right) \ominus L^{2}\left(D \rtimes_{\gamma^{2}} \mathbb{Z}\right)$ is a multiple of the regular representation of $\Gamma_{0}$. On the other hand, by condition 4 in 4.1, the unitary representation $\Gamma_{0} \curvearrowright L^{2}\left(\pi_{e} \Gamma_{0}(B) \otimes D\right)$ given by $\left(\alpha_{g}^{1}\right)_{g \in \Gamma_{0}}$ is disjoint from the regular representation of $\Gamma_{0}$. Combining both observations, it follows that $\psi\left(\pi_{e} \Gamma_{0}(B) \otimes D\right) \subset$ $D \rtimes_{\gamma^{2}} \mathbb{Z}$. Since $\psi \circ \alpha_{g}^{1}=\alpha_{\delta(g)}^{2} \circ \psi$ for all $g \in \Gamma$ and using (4.1), we arrive at the contradiction that $\psi\left(N_{1}\right) \subset D \rtimes_{\gamma^{2}} \mathbb{Z}$. So there indeed exists a $g_{0} \in \Gamma$ such that $g_{0} \delta\left(\Gamma_{0}\right) g_{0}^{-1} \cap \Gamma_{0} \neq\{e\}$.

After replacing $\psi$ by $\alpha_{g_{0}}^{2} \circ \psi$ and $\delta$ by $\left(\operatorname{Ad} g_{0}\right) \circ \delta$, we may assume that $\delta\left(\Gamma_{0}\right) \cap$ $\Gamma_{0} \neq\{e\}$ and we find $g_{1} \in \Gamma_{0}$ with $g_{1} \neq e$ and $\delta\left(g_{1}\right) \in \Gamma_{0}$. Since $\Gamma_{0}$ is abelian, it follows that $\delta^{-1}\left(\Gamma_{0}\right)$ commutes with $g_{1}$. By malnormality of $\Gamma_{0}<\Gamma$, we conclude that $\delta^{-1}\left(\Gamma_{0}\right) \subset \Gamma_{0}$. Applying $\delta$, it follows that $\Gamma_{0} \subset \delta\left(\Gamma_{0}\right)$. Since $\delta\left(\Gamma_{0}\right)$ is abelian and $\Gamma_{0}<\Gamma$ is malnormal, we find that $\Gamma_{0}=\delta\left(\Gamma_{0}\right)$.

For $i=1,2$, define $K_{i}=L^{2}\left(\left(\pi_{e} \Gamma_{0}(B) \bar{\otimes} D\right) \rtimes_{\gamma^{i}} \mathbb{Z}\right)$ and $L_{i}=L^{2}\left(N_{i}\right) \ominus K_{i}$. Since $\Gamma_{0}<\Gamma$ is malnormal, it follows from Lemma 3.2 that the unitary representation $\left(\alpha_{\delta(g)}^{2}\right)_{g \in \Gamma_{0}}$ of $\Gamma_{0}$ on $L_{2}$ is a multiple of the regular representation of $\Gamma_{0}$. By condition 4 in 4.1 , the unitary representation $\left(\alpha_{g}^{1}\right)_{g \in \Gamma_{0}}$ of $\Gamma_{0}$ on $K_{1}$ is disjoint from the regular representation of $\Gamma_{0}$. Combining both observations, it follows that $\psi\left(K_{1}\right) \subset K_{2}$. By symmetry, also the converse inclusion holds. So

$$
\psi\left(\left(\pi_{e \Gamma_{0}}(B) \bar{\otimes} D\right) \rtimes_{\gamma^{1}} \mathbb{Z}\right)=\left(\pi_{e \Gamma_{0}}(B) \bar{\otimes} D\right) \rtimes_{\gamma^{2}} \mathbb{Z} .
$$

We therefore find the isomorphism

$$
\Psi:(B \bar{\otimes} D) \rtimes_{\gamma^{1}} \mathbb{Z} \rightarrow(B \bar{\otimes} D) \rtimes_{\gamma^{2}} \mathbb{Z}
$$


satisfying $\left(\pi_{e \Gamma_{0}} \otimes \mathrm{id}\right) \circ \Psi=\psi \circ\left(\pi_{e \Gamma_{0}} \otimes \mathrm{id}\right)$. Because of (4.1) and the facts that $\psi(A \bar{\otimes} D)=A \bar{\otimes} D$ and $\psi(D)=D$, we have

$$
\Psi(B \bar{\otimes} D)=B \bar{\otimes} D \quad, \quad \Psi\left(D \rtimes_{\gamma^{1}} \mathbb{Z}\right)=D \rtimes_{\gamma^{2}} \mathbb{Z} \quad \text { and } \quad \Psi(D)=D .
$$

Since $D=L^{\infty}(Z, \eta)$, we obtain the nonsingular automorphism $\theta \in \operatorname{Aut}(Z, \eta)$ satisfying $\Psi(d)=d \circ \theta^{-1}$ for all $d \in L^{\infty}(Z, \eta)$. Since $\Psi\left(D \rtimes_{\gamma^{1}} \mathbb{Z}\right)=D \rtimes_{\gamma^{2}} \mathbb{Z}$, the map $\theta$ is an orbit equivalence between the essentially free actions $\gamma^{i}: \mathbb{Z} \curvearrowright(Z, \eta)$. So we find the 1-cocycle $\omega: \mathbb{Z} \times Z \rightarrow \mathbb{Z}$ satisfying $\theta(n \cdot z)=\omega(n, z) \cdot \theta(z)$ for all $n \in \mathbb{Z}$ and a.e. $z \in Z$. For all $n, m \in \mathbb{Z}$, denote by $p_{n, m} \in L^{\infty}(Z)$ the projection with support $\left\{z \in Z \mid \omega\left(n, \theta^{-1}(z)\right)=m\right\}$. Denote by $\left(u_{n}\right)_{n \in \mathbb{Z}}$ the canonical unitaries in the crossed product $D \rtimes_{\gamma^{i}} \mathbb{Z}$. Then,

$$
\Psi\left(u_{n}\right)=\sum_{m \in \mathbb{Z}} u_{m} \mu_{n, m}
$$

for all $n \in \mathbb{Z}$, where $\mu_{n, m} \in \mathcal{U}\left(D p_{n, m}\right)$.

We identify $B \bar{\otimes} D=L^{\infty}(Z, B)$. Since the automorphism $\Psi \in \operatorname{Aut}(B \bar{\otimes} D)$ satisfies $\Psi(D)=D$, we find a measurable family of automorphisms $\psi_{z} \in \operatorname{Aut}(B)$, for a.e. $z \in Z$, such that

$$
(\Psi(b))(\theta(z))=\psi_{z}(b(z)) \text { for all } b \in L^{\infty}(Z, B) \text { and a.e. } z \in Z \text {. }
$$

The isomorphism $\psi$ scales the trace by a scaling factor $\lambda>0$. Thus $\theta$ scales the measure by the same factor $\lambda$ and for a.e. $z \in Z$, the automorphism $\psi_{z}$ is trace preserving.

For all $n \in \mathbb{Z}$ and $b \in B$, we have

$$
\Psi\left(\gamma_{-n}(b) \otimes 1\right)=\Psi\left(u_{n}^{*}(b \otimes 1) u_{n}\right)=\Psi\left(u_{n}\right)^{*} \Psi(b \otimes 1) \Psi\left(u_{n}\right) .
$$

The left and right hand side both belong to $L^{\infty}(Z, B)$. Evaluating the left and right hand side in a point $\theta(z)$ for some $z \in Z$ with $\omega(n, z)=m$ and using (4.2), we obtain the equality

$$
\psi_{z}\left(\gamma_{-n}(b)\right)=\gamma_{-m}\left(\psi_{n \cdot z}(b)\right) .
$$

It follows that

$$
\psi_{n \cdot z} \circ \gamma_{n}=\gamma_{\omega(n, z)} \circ \psi_{z}
$$

for all $n \in \mathbb{Z}$ and a.e. $z \in Z$.

Since $\psi \circ \alpha_{g}^{1}=\alpha_{\delta(g)}^{2} \circ \psi$ for all $g \in \Gamma$, it follows that $\Psi \circ \beta_{g}=\beta_{\delta(g)} \circ \Psi$ for all $g \in \Gamma_{0}$. This means that $\psi_{z} \circ \beta_{g}=\beta_{\delta(g)} \circ \psi_{z}$ for all $g \in \Gamma_{0}$ and a.e. $z \in Z$. By conditions 2 and 3 in 4.1, it follows that $\delta(g)=g$ for all $g \in \Gamma_{0}$ and that a.e. $\psi_{z}$ is given by a power of $T$. So we find a measurable map $\varphi: Z \rightarrow \mathbb{Z}$ such that $\psi_{z}=\gamma_{\varphi(z)}$ for a.e. $z \in Z$.

Writing $\omega^{\prime}(n, z)=\varphi(n \cdot z)^{-1} \omega(n, z) \varphi(z)$, it then follows from (4.3) that $\gamma_{\omega^{\prime}(n, z)}=\gamma_{n}$. We conclude that $\omega^{\prime}(n, z)=n$ for all $n \in \mathbb{Z}$ and a.e. $z \in Z$. It 
follows that the map $z \mapsto \varphi(z)^{-1} \cdot \theta(z)$ is an automorphism of $(Z, \eta)$. Denoting, for all $m \in \mathbb{Z}$, by $q_{m} \in L^{\infty}(Z)$ the projection with support $\left\{z \in Z \mid \varphi\left(\theta^{-1}(z)\right)=m\right\}$, it follows that

$$
U=\sum_{m \in \mathbb{Z}} q_{m} u_{m}
$$

is a well defined unitary operator in $D \rtimes_{\gamma^{2}} \mathbb{Z}$. Replacing $\psi$ by $\left(\operatorname{Ad} U^{*}\right) \circ \psi$, we get that

$$
\psi\left(\left(\pi_{e \Gamma_{0}}(b) \otimes d\right) u_{n}\right)=\left(\pi_{e \Gamma_{0}}(b) \otimes \theta(d)\right) u_{n}
$$

for all $b \in B, d \in D, n \in \mathbb{Z}$, where $\theta \in \operatorname{Aut}(D)$ satisfies $\theta \circ \gamma_{n}^{1}=\gamma_{n}^{2} \circ \theta$ for all $n \in \mathbb{Z}$. We still have that $\psi \circ \alpha_{g}^{1}=\alpha_{\delta(g)}^{2} \circ \psi$ for all $g \in \Gamma$, where $\delta \in \operatorname{Aut}(\Gamma)$ satisfies $\delta(g)=g$ for all $g \in \Gamma_{0}$.

So $\psi$ is indeed the composition of the two isomorphisms described in the statement of the theorem.

Remark 4.3. Fix the same actions $\Gamma \curvearrowright(X, \mu)$ and $\mathbb{Z} \curvearrowright(X, \mu)$ as in Theorem 4.2. Whenever $\left(\eta_{n}\right)_{n \in \mathbb{Z}}$ is an outer action of $\mathbb{Z}$ on the hyperfinite $\mathrm{II}_{1}$ factor $R$, we consider, in the same way as in Theorem 4.2, the action $\left(\alpha_{g}^{\eta}\right)_{g \in \Gamma}$ of $\Gamma$ on

$$
\left(L^{\infty}(X) \bar{\otimes} R\right) \rtimes_{\eta} \mathbb{Z} .
$$

Contrary to the situation in Theorem 4.2, where we use the abelian algebra $L^{\infty}(Z)$ instead of $R$, this construction is of little interest since all the actions $\left(\alpha_{g}^{\eta}\right)_{g \in \Gamma}$ are isomorphic. Indeed, take two outer actions $\left(\eta_{n}\right)_{n \in \mathbb{Z}}$ and $\left(\eta_{n}^{\prime}\right)_{n \in \mathbb{Z}}$ of $\mathbb{Z}$ on $R$. By [7, Theorem 2], the automorphisms $\eta_{1}$ and $\eta_{1}^{\prime}$ are outer conjugate. So we find $\psi_{0} \in \operatorname{Aut}(R)$ and a unitary $v_{1} \in \mathcal{U}(R)$ such that $\psi_{0} \circ \eta_{1}=\operatorname{Ad} v_{1} \circ \eta_{1}^{\prime} \circ \psi_{0}$. Denoting by $\left(u_{n}\right)_{n \in \mathbb{Z}}$ the canonical generating unitaries of $L(\mathbb{Z})$, one checks that there is a unique isomorphism

$$
\psi:\left(L^{\infty}(X) \bar{\otimes} R\right) \rtimes_{\eta} \mathbb{Z} \rightarrow\left(L^{\infty}(X) \bar{\otimes} R\right) \rtimes_{\eta^{\prime}} \mathbb{Z}
$$

satisfying $\psi(a \otimes b)=a \otimes \psi_{0}(b)$ for all $a \in L^{\infty}(X), b \in R$ and $\psi\left(u_{1}\right)=\left(1 \otimes v_{1}\right) u_{1}$. By construction, $\psi \circ \alpha_{g}^{\eta}=\alpha_{g}^{\eta^{\prime}} \circ \psi$ for all $g \in \Gamma$.

We are now ready to prove Theorem A.

Proof of Theorem A. We apply Theorem 4.2 to the group $\Gamma=(\mathbb{Z} / n \mathbb{Z}) *(\mathbb{Z} / m \mathbb{Z})$. Denote by $a \in \mathbb{Z} / n \mathbb{Z}$ and $b \in \mathbb{Z} / m \mathbb{Z}$ the cyclic generators. Define $\Gamma_{0} \cong \mathbb{Z}$ as the subgroup of $\Gamma$ generated by $a b$. By [6, Example 7.C], $\Gamma_{0}$ is a malnormal subgroup of $\Gamma$. For every ergodic measure preserving automorphism $\Delta$ of a standard nonatomic finite or infinite measure space $(Z, \eta)$, Theorem 4.2 provides the outer action $\left(\alpha_{g}^{\Delta}\right)_{g \in \Gamma}$ of $\Gamma$ on $N=L^{\infty}(X \times Z) \rtimes \mathbb{Z}$.

To prove the first statement of Theorem A, take $H \in \mathcal{S}$. By [1, Theorem 4.3], there exists an ergodic measure preserving automorphism $\Delta$ of a standard infinite 
measure space $(Z, \eta)$ such that

$$
H=\{\bmod (\theta) \mid \theta \in \operatorname{Aut}(Z, \eta) \text { commutes with } \Delta\} .
$$

Fix a nonzero projection $p \in L^{\infty}(Z)$ of finite trace and realize the hyperfinite $\mathrm{II}_{1}$ factor $R$ as $p N p$. We still denote by $\alpha^{\Delta}$ the restriction of $\alpha^{\Delta}$ to $R=p N p$. We consider the associated group-type subfactor $S\left(\alpha^{\Delta}\right)$. We claim that $\mathcal{F}\left(S\left(\alpha^{\Delta}\right)\right)=H$.

First assume that $\lambda \in \mathcal{F}\left(S\left(\alpha^{\Delta}\right)\right)$. By [4, Theorem 3.2], we find a projection $q \in L^{\infty}(Z)$ with $\operatorname{Tr}(q)=\lambda \operatorname{Tr}(p)$ and an isomorphism $\psi: p N p \rightarrow q N q$ such that $\psi$ is an outer conjugacy between the restrictions of $\alpha^{\Delta}$ to $p N p$, resp. $q N q$. We can then amplify $\psi$ to an outer conjugacy $\psi \in \operatorname{Aut}(N)$ of $\alpha^{\Delta}$ scaling the trace by the module $\lambda$. Combining the remarks after Theorem 4.2 with formula (4.4), we conclude that $\lambda \in H$.

Conversely, if $\lambda \in H$, we find by (4.4) an automorphism $\psi \in \operatorname{Aut}(N)$ that commutes with the action $\alpha^{\Delta}$ and that scales the trace by the module $\lambda$. Put $q=\psi(p)$. Then $q$ is an $\alpha^{\Delta}$-invariant projection in $N$ with $\operatorname{Tr}(q)=\lambda \operatorname{Tr}(p)$ and $\psi$ induces an isomorphism between the subfactors

$$
(p N p)^{\mathbb{Z} / n \mathbb{Z}} \subset(p N p) \rtimes \mathbb{Z} / m \mathbb{Z} \quad \text { and } \quad(q N q)^{\mathbb{Z} / n \mathbb{Z}} \subset(q N q) \rtimes \mathbb{Z} / m \mathbb{Z} .
$$

This precisely means that $\lambda \in \mathcal{F}\left(S\left(\alpha^{\Delta}\right)\right)$.

To prove the second statement of Theorem A, we take for $(Z, \eta)$ a standard nonatomic probability space. For every ergodic pmp automorphism $\Delta \in \operatorname{Aut}(Z, \eta)$, Theorem 4.2 provides an outer action $\left(\alpha_{g}^{\Delta}\right)_{g \in \Gamma}$ on the hyperfinite $\mathrm{II}_{1}$ factor $R=L^{\infty}(X \times Z) \rtimes \mathbb{Z}$. We denote by $S\left(\alpha^{\Delta}\right)$ the associated group-type subfactor.

If the subfactors $S\left(\alpha^{\Delta_{1}}\right)$ and $S\left(\alpha^{\Delta_{2}}\right)$ are isomorphic, it follows from [4, Theorem 3.2] that the actions $\alpha^{\Delta_{1}}$ and $\alpha^{\Delta_{2}}$ are outer conjugate. It then follows from Theorem 4.2 that $\Delta_{1}, \Delta_{2}$ are conjugate inside $\operatorname{Aut}(Z, \eta)$. Conversely, when $\Delta_{1}, \Delta_{2}$ are conjugate inside $\operatorname{Aut}(Z, \eta)$, the actions $\alpha^{\Delta_{1}}, \alpha^{\Delta_{2}}$ are isomorphic and hence, the subfactors $S\left(\alpha^{\Delta_{1}}\right), S\left(\alpha^{\Delta_{2}}\right)$ are isomorphic.

Finally note that by [2, Theorem 5.1], the standard invariant of the subfactor $R^{H} \subset R \rtimes K$ only depends on the inclusions $H, K \subset \Gamma$.

As explained in the introduction, we also provide as a corollary of Theorem 4.2, the following new explicit construction of $\mathrm{II}_{1}$ factors with a prescribed fundamental group in the family $\mathcal{S}$ of [31, Section 2].

Corollary 4.4. Let $\Gamma$ be a nonamenable, weakly amenable, bi-exact, icc group containing a copy of $\mathbb{Z}$ as a malnormal subgroup $\Gamma_{0}<\Gamma$, e.g. take $\Gamma=\mathbb{F}_{2}=$ $\mathbb{Z} * \mathbb{Z}$ with $\Gamma_{0}$ given by the first copy of $\mathbb{Z}$. For every ergodic measure preserving automorphism $\Delta$ of a standard infinite measure space $(Z, \eta)$, consider the action $\left(\alpha_{g}^{\Delta}\right)_{g \in \Gamma}$ of $\Gamma$ on $N=L^{\infty}(X \times Z) \rtimes \mathbb{Z}$ as in Theorem 4.2. Fix a projection $p \in L^{\infty}(Z)$ with $\operatorname{Tr}(p)<\infty$.

The fundamental group of the $I_{1}$ factor $p N p \rtimes \Gamma$ is given by $\bmod \left(\operatorname{Centr}_{\mathrm{Aut}(Z, \eta)}(\Delta)\right)$. 
Proof. First assume that $\lambda>0$ belongs to the fundamental group of $p N p \rtimes \Gamma$. We can then take a projection $q \in L^{\infty}(Z)$ with $\operatorname{Tr}(q)=\lambda \operatorname{Tr}(p)$ and an isomorphism $\psi: p N p \rtimes \Gamma \rightarrow q N q \rtimes \Gamma$. By [33, Theorem 1.4], we have $\psi(p N p) \prec q N q$ and $q N q \prec \psi(p N p)$. It then follows from [17, Lemma 8.4] that $\psi(p N p)$ and $q N q$ are unitarily conjugate. So $\psi$ defines a cocycle conjugacy of the action $\left(\alpha_{g}^{\Delta}\right)_{g \in \Gamma}$ scaling the trace by the module $\lambda$. By Theorem 4.2, we have that $\lambda=\bmod (\theta)$ for some $\theta \in \operatorname{Aut}(Z, \eta)$ that commutes with $\Delta$.

Conversely, every $\theta \in \operatorname{Aut}(Z, \eta)$ that commutes with $\Delta$ defines an automorphism of $N$ that commutes with $\alpha^{\Delta}$ and hence extends to an automorphism of $N \rtimes \Gamma$ scaling the trace by the module $\lambda$. It follows that $\bmod (\theta)$ belongs to the fundamental group of $p N p \rtimes \Gamma$.

\section{References}

[1] J. Aaronson, The intrinsic normalising constants of transformations preserving infinite measures. J. Analyse Math. 49 (1987), 239-270. Zbl 0644.28013 MR 928513

[2] D. Bisch, P. Das and S. K. Ghosh, The planar algebra of group-type subfactors. J. Funct. Anal. 257 (2009), 20-46. Zbl 1172.46042 MR 2523334

[3] D. Bisch and U. Haagerup, Composition of subfactors: new examples of infinite depth subfactors. Ann. Sci. École Norm. Sup. (4) 29 (1996), 329-383. Zbl 0853.46062 MR 1386923

[4] D. Bisch, R. Nicoara and S. Popa, Continuous families of hyperfinite subfactors with the same standard invariant. Internat. J. Math. 18 (2007), 255-267. Zbl 1120.46047 MR 2314611

[5] J. Bourgain, On the spectral type of Ornstein's class one transformations. Israel J. Math. 84 (1993), 53-63. Zbl 0787.28011 MR 1244658

[6] P. de la Harpe and C. Weber, Malnormal subgroups and Frobenius groups: basics and examples. With an appendix by D. Osin. Confluentes Math. 6 (2014), 65-76. Zbl 06421327 MR 3266885

[7] A. Connes, Outer conjugacy classes of automorphisms of factors. Ann. Sci. École Norm. Sup. (4) 8 (1975), 383-419. Zbl 0342.46052 MR 394228

[8] A. del Junco and D. Rudolph, On ergodic actions whose self-joinings are graphs. Ergodic Theory Dynam. Systems 7 (1987), 531-557. Zbl 0646.60010 MR 922364

[9] S. Deprez, Explicit examples of equivalence relations and factors with prescribed fundamental group and outer automorphism group. Trans. Amer. Math. Soc. 367 (2015), 6837-6876. Zbl 06479343 MR 3378816 
[10] I. Epstein, Orbit inequivalent actions of non-amenable groups. Preprint. arXiv:0707.4215, 2008.

[11] M. Foreman, D. J. Rudolph and B. Weiss, The conjugacy problem in ergodic theory. Ann. of Math. 173 (2011), 1529-1586. Zbl 1243.37006 MR 2800720

[12] D. Gaboriau and R. Lyons, A measurable-group-theoretic solution to von Neumann's problem. Invent. Math. 177 (2009), 533-540. Zbl 1182.43002 MR 2534099

[13] D. Gaboriau and S. Popa, An uncountable family of nonorbit equivalent actions of $\mathbb{F}_{n}$. J. Amer. Math. Soc. 18 (2005), 547-559. Zbl 1155.37302 MR 2138136

[14] G. Hjorth, On invariants for measure preserving transformations. Fund. Math. 169 (2001), 51-84. Zbl 0990.03036 MR 1852353

[15] A. Ioana, Rigidity results for wreath product $\mathrm{II}_{1}$ factors. J. Funct. Anal. 252 (2007), 763-791. Zbl 1134.46041 MR 2360936

[16] A. Ioana, Orbit inequivalent actions for groups containing a copy of $\mathbb{F}_{2}$. Invent. Math. 185 (2011), 55-73. Zbl 1230.37010 MR 2810796

[17] A. Ioana, J. Peterson and S. Popa, Amalgamated free products of weakly rigid factors and calculation of their symmetry groups. Acta Math. 200 (2008), 85153. Zbl 1149.46047 MR 2386109

[18] V. F. R. Jones, Index for subfactors. Invent. Math. 72 (1983), 1-25. Zbl 0508.46040 MR 696688

[19] V. F. R. Jones, A converse to Ocneanu's theorem. J. Operator Theory 10 (1983), 61-63. Zbl 0547.46045 MR 715556

[20] A. Ocneanu, Actions of discrete amenable groups on von Neumann algebras. Lecture Notes in Mathematics 1138, Springer-Verlag, Berlin, 1985. Zbl 0608.46035 MR 807949

[21] D. Ornstein, On the root problem in ergodic theory. In Proceedings of the Sixth Berkeley Symposium on Mathematical Statistics and Probability 1970/1971, vol. II, pp. 347-356, Univ. California Press, Berkeley, 1972. Zbl 0262.28009 MR 399415

[22] D. Ornstein and B. Weiss, Ergodic theory of amenable group actions, I. Bull. Amer. Math. Soc. (N.S.) 2 (1980), 161-164. Zbl 0427.28018 MR 551753

[23] S. Popa, Maximal injective subalgebras in factors associated with free groups. Adv. Math. 50 (1983), 27-48. Zbl 0545.46041 MR 720738

[24] S. Popa, Relative dimension, towers of projections and commuting squares of subfactors. Pac. J. Math. 137 (1989), 181-207. Zbl 0699.46042 MR 983336

[25] S. Popa, Classification of amenable subfactors of type II. Acta Math. 172 (1994), 163-255. Zbl 0853.46059 MR 1278111 
[26] S. Popa, Some rigidity results for non-commutative Bernoulli shifts. J. Funct. Anal. 230 (2006), 273-328. Zbl 1097.46045 MR 2186215

[27] S. Popa, On a class of type $\mathrm{II}_{1}$ factors with Betti numbers invariants. Ann. of Math. 163 (2006), 809-899. Zbl 1120.46045 MR 2215135

[28] S. Popa, Strong rigidity of $\mathrm{II}_{1}$ factors arising from malleable actions of $w$-rigid groups, I. Invent. Math. 165 (2006), 369-408. Zbl 1120.46043 MR 2231961

[29] S. Popa, On the superrigidity of malleable actions with spectral gap. J. Amer. Math. Soc. 21 (2008), 981-1000. Zbl 1222.46048 MR 2425177

[30] S. Popa and S. Vaes, Strong rigidity of generalized Bernoulli actions and computations of their symmetry groups. Adv. Math. 217 (2008), 833-872. Zbl 1137.37003 MR 2370283

[31] S. Popa and S. Vaes, Actions of $\mathbb{F}_{\infty}$ whose $\mathrm{II}_{1}$ factors and orbit equivalence relations have prescribed fundamental group. J. Amer. Math. Soc. 23 (2010), 383-403. Zbl 1202.46069 MR 2601038

[32] S. Popa and S. Vaes, Unique Cartan decomposition for $\mathrm{II}_{1}$ factors arising from arbitrary actions of free groups. Acta Math. 212 (2014), 141-198. Zbl 1307.46047 MR 3179609

[33] S. Popa and S. Vaes, Unique Cartan decomposition for $\mathrm{II}_{1}$ factors arising from arbitrary actions of hyperbolic groups. J. Reine Angew. Math. 694 (2014), 215239. Zbl 06344257 MR 3259044

[34] D. J. Rudolph, An example of a measure preserving map with minimal self-joinings, and applications. J. Analyse Math. 35 (1979), 97-122. Zbl 0446.28018 MR 555301

[35] S. Vaes, Rigidity results for Bernoulli actions and their von Neumann algebras (after Sorin Popa). Séminaire Bourbaki, exp. no. 961. Astérisque 311 (2007), 237-294. Zbl 1194.46085 MR 2359046

[36] S. Vaes, Explicit computations of all finite index bimodules for a family of $\mathrm{II}_{1}$ factors. Ann. Sci. Éc. Norm. Supér. 41 (2008), 743-788. Zbl 1194.46086 MR 2504433

Received 04 October, 2013

A. Brothier, Vanderbilt University, Department of Mathematics, 1326 Stevenson Center Ln, Nashville, TN 37235, USA

E-mail: arnaud.brothier@vanderbilt.edu

S. Vaes, KU Leuven, Department of Mathematics, Celestijnenlaan 200B, Leuven 3001, Belgium

E-mail: stefaan.vaes@wis.kuleuven.be 